\title{
Influence of thermal fluctuations on quantum phase transitions in one-dimensional disordered systems: Charge density waves and Luttinger liquids
}

\author{
Andreas Glatz ${ }^{1,2}$ and Thomas Nattermann ${ }^{1}$ \\ ${ }^{1}$ Institut für Theoretische Physik, Universität zu Köln, Zülpicher Str. 77, 50937 Köln, Germany \\ ${ }^{2}$ Materials Science Division, Argonne National Laboratory, Argonne, Illinois 60439
}

(Dated: October 29, 2018)

\begin{abstract}
The low temperature phase diagram of 1D weakly disordered quantum systems like charge or spin density waves and Luttinger liquids is studied by a full finite temperature renormalization group (RG) calculation. For vanishing quantum fluctuations this approach is amended by an exact solution in the case of strong disorder and by a mapping onto the Burgers equation with noise in the case of weak disorder, respectively. At zero temperature we reproduce the quantum phase transition between a pinned (localized) and an unpinned (delocalized) phase for weak and strong quantum fluctuations, respectively, as found previously by Fukuyama or Giamarchi and Schulz.

At finite temperatures the localization transition is suppressed: the random potential is wiped out by thermal fluctuations on length scales larger than the thermal de Broglie wave length of the phason excitations. The existence of a zero temperature transition is reflected in a rich cross-over phase diagram of the correlation functions. In particular we find four different scaling regions: a classical disordered, a quantum disordered, a quantum critical and a thermal region. The results can be transferred directly to the discussion of the influence of disorder in superfluids. Finally we extend the RG calculation to the treatment of a commensurate lattice potential. Applications to related systems are discussed as well.
\end{abstract}

PACS numbers: $71.10 . \mathrm{Pm}, 72.15 . \mathrm{Rn} / \mathrm{Nj}, 73.20 . \mathrm{Mp} / \mathrm{Jc}$

\section{INTRODUCTION}

The collective behavior of condensed modulated structures like charge or spin density waves (CDWs/SDWs) ${ }^{1.2 .3}$, flux line lattices ${ }^{4.5}$ and Wigner crystals $\frac{3}{3}$ in random environments has been the subject of detailed investigations since the early 1970s. These were motivated by the drastic influence of disorder: without pinning CDWs would be ideal superconductors whereas type-II superconductors would show finite resistivity. In three dimensional systems the low temperature phase of these structures is determined by a zero temperature disorder fixed point resulting in quasi-long-range order and glassy dynamics (for recent reviews and further references see 4,5]). In two dimensions this fixed point is extended to a fixed line which terminates at the glass transition temperature ${ }^{6.7}$. In the low temperature phase, correlations of the positional order decay slightly faster than a power law and the linear resistivity vanishes 5 .

In one dimension the situation is different: the glass temperature is shifted to $T=0$. Nevertheless, there remains a residual trace of disorder which is reflected in the low temperature behavior of spatial correlations and the dynamics $\frac{8.9}{}$. Clearly, at low temperatures also quantum fluctuations have to be taken into account. Disorder and quantum fluctuations in $1 \mathrm{D}$ CDWs at zero temperature have been considered previously (see, e.g., 1011]) and an unpinning (delocalization) transition as a function of the strength of quantum fluctuations was found. Finite temperature effects were partially incorporated by truncating the renormalization group ( $R G$ ) flow at the de Broglie wave length of the phason excitations 11. However, for a complete study of the thermal to quantum crossover, quantum and thermal fluctuations have to be considered on an equal footing ${ }^{12}$, which is the main aim of this paper.

Experimentally, quasi-1D behavior can be seen in real materials, e.g., in whiskers ${ }^{13}$, hairlike single crystal fibers like $\mathrm{NbSe}_{3}$, with a transverse extension smaller than the correlation length or in chain like crystals with weak interchain coupling. In the latter case there is a large crossover length scale up to which $1 \mathrm{D}$ behavior can be observed 1.3 .

The results obtained for the CDWs or SDWs have a large number of further applications on disordered quantum systems: they relate, e.g., to the localization transition of Luttinger liquids ${ }^{10.11}$, superfluids ${ }^{14}$, tunnel junction chains $\frac{15}{5}$, Josephson coupled chains of these systems, if the coupling is treated in mean-field theory $\stackrel{10}{ }$, and CDWs in a lattice potential. However, we will use the terminology of CDWs in most parts of this paper.

The remaining part of the paper is organized as follows: In section 1 we give a detailed introduction to our model and the notation used in this paper. We also briefly discuss the influence of Coulomb interaction on the properties of the system. In section ஹthe influence of the disorder is studied in detail. Using an anisotropic momentum shell renormalization group calculation, in which the full Matsubara sum over frequncies is performed, we obtain flow equations for the effective strength of the disorder, thermal and quantum fluctuations (i.e., the interaction strength in the case of Luttinger liquids). These are discussed first in the case of zero temperature and agreement with previous results is obtained ${ }^{10.11}$. At finite temperatures the disorder always renormalizes to zero. In the classical limit two more methods are applied: (i) 
at low temperatures and strong disorder the ground state of the model is calculated exactly. (ii) For weak disorder and strong thermal fluctuations a second RG calculation, based on the mapping onto the Burgers equation with noise, is applied. Using all these findings, the phase diagram of the density-density correlation function is studied in section IV The main result of this section is the calculation of the low temperature quantum crossover diagram for one-dimensional CDWs. In the following section $\nabla$ we discuss briefly the application of the results to superfluids by using the mapping to CDWs. Some of these results were previously presented in Ref. 16.

The influence of a commensurate lattice potential on a free density wave is considered in section DI The full finite temperature renormalization group flow equation for this sine-Gordon type model are derived and resulting phase diagram is discussed.

In the appendices we present the calculation of the renormalization group flow equations and the derivation of the correlation function in the strong and weak pinning limit in some detail for the interested reader. In the final section of the appendix we list all symbols used in this paper with corresponding references in the paper.

\section{MODEL}

\section{A. charge and spin density}

In this section we derive the effective Hamiltonian which will be the starting point for our further treatment. The strategy of the calculation is therefore separated into two steps. In the first step the system is treated in a mean-field (MF) type approximation applied to a microscopic Hamiltonian. This leaves us with a slowly varying complex order parameter field for which we derive an effective Hamiltonian. The second step involves the consideration of the fluctuations of this order parameter, which is the topic of this paper.

We briefly summarize now the result of the mean-field calculation: Well below the mean-field condensation temperature $T_{M F}$ of the CDW, the underlying lattice will be periodically distorted with a period $\lambda$ which is related to the Fermi wave vector $k_{F}$ by $\lambda=\pi / k_{F}$. This distortion of the lattice leads to the formation of a gap in the dispersion relation at $k= \pm k_{F}$ which is (in one dimension) proportional to the amplitude of the lattice modulation. For small displacements (which are typically smaller than $1 \%$ of the interatomic spacing ${ }^{17}$ ), the increase of the elastic energy is smaller than the gain of electronic energy due to the formation of the gap and hence an instability is favored. The period of the CDW depends on the band filling factor (via $k_{F}=\pi / \lambda$ ) and is in general at arbitrary band filling incommensurate with the undistorted lattice (with lattice constant $a$ ).

In (quasi-)one-dimensional systems ${ }^{2}$ also SDWs can be found, but in contrast to CDWs they arise due to electron-electron and not to electron-phonon interaction.
A SDW can be considered to consist of two CDWs, one for spin-up and another for spin-down electrons (see, e.g., Fig. 5 in [2]). Therefore the spatial modulation of SDWs is characterized by a wave vector $Q=2 k_{F}$, as for CDWs.

The charge or spin density $\rho(x, t)$ can be written in the form 2.18

$\rho(x, t)=\left(1+Q^{-1} \partial_{x} \varphi(x, t)\right)\left[\rho_{0}+\rho_{1} \cos (p \varphi(x, t)+p Q x)\right]$

where $\rho_{0}=Q f(T) / \pi$ and $\rho_{1}=2|\Delta| /\left(\pi g v_{F}\right) . \quad g$ is the dimensionless electron-phonon coupling constant and $v_{F}$ the Fermi velocity. $\rho_{1}$ is proportional to $|\Delta|$, the CDW gap or the amplitude of the complex (mean field) order parameter

$$
\Delta(x, t)=|\Delta(x, t)| e^{\imath \varphi(x, t)} .
$$

$f$ is the condensate density $(Y=1-f$ is the so-called Yoshida function) related to $|\Delta|$ by

$$
f(T)=\frac{\pi T}{\hbar} \sum_{\omega_{n}} \frac{(|\Delta| / \hbar)^{2}}{\left(\omega_{n}^{2}+(|\Delta| / \hbar)^{2}\right)^{3 / 2}}, \quad \omega_{n}=2 \pi n T / \hbar .
$$

The condensate density approaches 1 for $T \rightarrow 0$ and $f(T) \simeq 2\left(1-T / T_{c}^{M F}\right) \sim|\Delta|^{2}$ for $T \rightarrow T_{c}^{M F} . T_{c}^{M F}$ denotes the mean-field transition temperature. For quasi one-dimensional systems $\rho_{1}$ has an additional factor $\zeta^{-2}$ (the area perpendicular to the chain).

Note, that (11) is correct for arbitrary band filling and, to be more precisely, is the particle density of the charge or spin carrying particles. The particle current density $j$ follows from (2) as $j=-\rho_{0} \dot{\varphi} / Q$.

Because $4 k_{F}$ modulations of SDWs or CDWs are also possible $\frac{19}{}$, we introduce the factor $p$ in the argument of the modulating cosine function, i.e., for CDWs and SDWs $p$ is usually 1 , but can also be 2 or greater.

\section{B. Hamiltonian}

In the following we use a minimal model for the low energy, long wave length excitations of the condensed charge density wave. Since fluctuations in the amplitude $|\Delta|$ are suppressed, because these are massive, we take into account only fluctuations in the phase $\varphi$. Clearly, such an approach breaks down sufficiently close to the mean-field transition temperature $T_{c}^{M F}$. Neglecting fluctuations in $|\Delta|$, the Hamiltonian of the CDW is given by

$$
\hat{\mathcal{H}}=\hat{\mathcal{H}}_{0}+\hat{\mathcal{H}}_{u}+\hat{\mathcal{H}}_{w}
$$


with

$$
\begin{aligned}
& \hat{\mathcal{H}}_{0} \equiv \int_{0}^{L} d x \frac{c}{2}\left[\left(\frac{v}{c}\right)^{2} \hat{P}^{2}+\left(\partial_{x} \hat{\varphi}\right)^{2}\right], \\
& \hat{\mathcal{H}}_{u} \equiv \int_{0}^{L} d x U(x) \rho(x), U(x)=\sum_{i=1}^{N_{\text {imp }}} U_{i} \delta\left(x-x_{i}\right)(4 \mathrm{~b}) \\
& \hat{\mathcal{H}}_{w} \equiv-\int_{0}^{L} d x W \cos (q \hat{\varphi}(x)) .
\end{aligned}
$$

$\hat{\mathcal{H}}_{0}$ describes the phason excitations of the CDW, where $c=\frac{\hbar v_{F}}{2 \pi} f(T)$ denotes the elastic constant. $v=$ $v_{F} / \sqrt{1+\left(2|\Delta| / \hbar \omega_{p Q}\right)^{2} /(g f)}$ is the effective velocity of the phason excitations with $\omega_{p Q}$ the phonon frequency. For CDWs $\left(2|\Delta| / \hbar \omega_{p Q}\right)^{2} /(g f) \gg 1$ is typically fulfilled and hence quantum fluctuations are weak.

$\hat{P}$ is the momentum operator, corresponding to the phase $\hat{\varphi}$, with the standard commutation relation $\left[\hat{P}(x), \hat{\varphi}\left(x^{\prime}\right)\right]=\frac{\hbar}{i} \delta\left(x-x^{\prime}\right)$

$\hat{\mathcal{H}}_{u}$ results from the effects of impurities with random potential strength $U_{i}$ and positions $x_{i}$. The potential strength is characterized by $\overline{U_{i}}=0$ and $\overline{U_{i} U_{j}} \equiv U_{i m p}^{2} \delta_{i, j}$, and includes a forward and a backward scattering term proportional to $\rho_{0}$ and $\rho_{1}$, respectively. The disorder average of the impurity potential $U(x)$ follows then to be given by $\overline{U(x)}=0$ and

$$
\overline{U(x) U(y)}=\frac{U_{i m p}^{2}}{l_{i m p}} \delta(x-y) .
$$

We will further assume, that the mean impurity distance $l_{i m p}=L / N_{i m p}$ is large compared with the wave length of the CDW and, in most parts of the paper, that the disorder is weak, i.e.,

$$
1 \ll l_{i m p} Q \ll c Q /\left(U_{i m p} \rho_{1}\right) .
$$

In this case the Fukuyama-Lee length

$$
L_{F L}=\left(\frac{c \sqrt{l_{i m p}}}{U_{i m p} \rho_{1} p^{2}}\right)^{2 / 3}
$$

is large compared to the impurity distance $l_{i m p}$.

The third term in (4), $\mathcal{H}_{w}$, includes the influence of a harmonic lattice potential. This term will be discussed

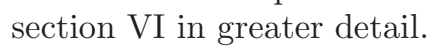

Our model (4I) includes the four dimensionless parameters

$$
\begin{aligned}
t & =T / \pi \Lambda c, \\
K & =\hbar v / \pi c, \\
u^{2} & =\frac{\left(U_{i m p} \rho_{1}\right)^{2}}{\Lambda^{3} \pi c^{2} l_{i m p}}, \\
w & =W / \pi c \Lambda^{2},
\end{aligned}
$$

which measure the strength of the thermal $(t)$, quantum $(K)$ and disorder fluctuations $(u)$ and the periodic potential $(w)$, respectively. $\Lambda=\pi / a$ is a momentum cutoff. The classical region of the model is given by $K \ll t$ which can be rewritten as the condition, that the thermal de Broglie wave length

$$
\lambda_{T}=\hbar \beta v=K /(t \Lambda)
$$

of the phason excitations is small compared to $a$.

At $T=0, K$-values of the order $10^{-2}$ to $10^{-1}$ and 1 , have been discussed for CDWs and SDWs, respectively ${ }^{1.20}$. It has to be noted however, that the the expressions relating $c$ and $v$ to the microscopic (mean-fieldlike) theory lead to the conclusion that $K$ and $t$ diverge by approaching $T_{c}^{M F}$, whereas the ratio $K / t$ remains finite.

\section{Coulomb Interaction}

We could also add a Coulomb interaction term to our model (4) which can be written as

$$
\hat{\mathcal{H}}_{c}=\frac{1}{2} \int d x \int d x^{\prime} \hat{\rho}(x) V_{c}\left(x-x^{\prime}\right) \hat{\rho}\left(x^{\prime}\right),
$$

where $V_{c}$ is the Coulomb potential. In all dimensions the unscreened potential has the form $e^{2} / r$. If we assume, that the quasi one-dimensional system has the finite width $\zeta, V_{c}$ can be written $\operatorname{as}^{21.22}$

$$
\begin{aligned}
V_{c}^{0}(x)= & \frac{e^{2}}{\sqrt{x^{2}+\zeta^{2}}}=\frac{1}{L} \sum_{k} e^{\imath k x} V_{c}^{0}(k) \text { with } \\
& V_{c}^{0}(k)=2 e^{2} K_{0}(|\zeta k|),
\end{aligned}
$$

where $K_{0}$ is a Bessel function with $K_{0}(x) \approx-\ln (x)$ for $x \ll 1$.

In general the Coulomb potential is screened and can be written as 23

$$
V_{c}(k, \omega)=\frac{V_{c}^{0}(k)}{1+V_{c}^{0}(k) \Pi(k, \omega),}
$$

with the momentum and frequency dependent polarization operator $\Pi(k, \omega)=\langle\rho(0,0) \rho(k, \omega)\rangle$.

If we only consider the static case $\omega=0$ we can distinguish two limiting cases: First, if the typical range $\lambda_{\text {eff }}$ of the screened Coulomb potential $V_{c}$ is much smaller than the mean electron distance, the potential can be assumed to be a delta distribution and $H_{c}$ can be approximated by

$$
\hat{\mathcal{H}}_{c} \approx \frac{\hbar \chi}{2} \int d x\left(\frac{1}{\pi} \partial_{x} \varphi(x)\right)^{2}+\ldots
$$

with $\chi=\frac{1}{\hbar} \int d x V_{c}(x)$. The cos-terms $(.$.$) from the den-$ sity can be neglected due to strong fluctuations. Therefore the Coulomb interaction gives only an additional 
contribution to the elastic constant of the initial model: $c=\frac{\hbar v_{F}}{2 \pi} f+\frac{\hbar \chi}{\pi^{2}}$. For $\chi>0$ the Coulomb interaction is repulsive, which leads to an increase of $c$ and therefore a decrease of the dimensionless parameter $K$, i.e., the quantum fluctuations will be reduced by the Coulomb interaction. In the case $\chi<0$ (attraction), $K$ will be increased. Keeping this consideration in mind, we will not further include $\hat{\mathcal{H}}_{c}$ in the model explicitly.

In the other case - with weak screening $-V_{c}(k) \approx$ $V_{c}^{0}(k)$ shows the dispersion given in (12) and in general, the details of the $k$-dependence depend not only on the transverse extension $\zeta$ of the quasi one-dimensional system under consideration but also on the screening length 14.21 .

However, the logarithmic $k$-dependence will only weakly affect our RG-analysis, but may suppress phase transitions, as discussed later in section IIIB

Coulomb interaction is also important if one considers multi-channel systems ${ }^{24}$ or the effect of the noncondensated normal electrons.

\section{RENORMALIZATION GROUP TREATMENT OF DISORDER}

\section{A. Flow equations}

In order to determine the phase diagram we adopt a standard Wilson-type renormalization group calculation, which starts from a path integral formulation of the partition function corresponding to the Hamiltonian (4). We begin with the renormalization of the disorder term and put $w=0$ in the following. The system is transformed into a translational invariant problem using the replica method, in which the disorder averaged free energy is calculated, using

$$
\overline{\mathcal{F}}=-T \overline{\ln \operatorname{Tr} e^{-\mathcal{S} / \hbar}} \equiv-T \lim _{n \rightarrow 0} \frac{1}{n}\left(\operatorname{Tr} e^{-\mathcal{S}^{(n)} / \hbar}-1\right),
$$

which defines the replicated action $\mathcal{S}^{(n)} \cdot \mathcal{S}^{(n)}$ is given by

$$
\mathcal{S}^{(n)}=\sum_{\alpha, \nu} \int_{\tau}\left\{\mathcal{L}_{0, \alpha} \delta_{\alpha \nu}+\frac{1}{2 \hbar} \int_{\tau^{\prime}} \overline{\mathcal{H}_{u}\left[\varphi_{\alpha}(\tau)\right] \mathcal{H}_{u}\left[\varphi_{\nu}\left(\tau^{\prime}\right)\right]}\right\}
$$

where $\mathcal{L}_{0}$ is the Lagrangian corresponding to $\hat{\mathcal{H}}_{0}, \int_{\tau} \equiv$ $\int_{0}^{\hbar \beta} d \tau$ and $\alpha, \nu$ are replica indices. Using (5) and consequently neglecting higher harmonics ( $2 p Q$-modes) one finds

$$
\overline{\mathcal{H}_{u}\left[\varphi_{\alpha}(\tau)\right] \mathcal{H}_{u}\left[\varphi_{\nu}\left(\tau^{\prime}\right)\right]}=\frac{U_{i m p}^{2} \rho_{1}^{2}}{2 l_{i m p}} \int_{0}^{L} d x\left\{\cos p\left(\varphi_{\alpha}(x, \tau)-\varphi_{\nu}\left(x, \tau^{\prime}\right)\right)+\frac{2 \rho_{0}^{2}}{Q^{2} \rho_{1}^{2}} \partial_{x} \varphi_{\alpha}(x, \tau) \partial_{x} \varphi_{\nu}\left(x, \tau^{\prime}\right)\right\}
$$

Together with (16) one obtains the following form

$$
\begin{gathered}
\frac{\mathcal{S}^{(n)}}{\hbar}=\frac{1}{2 \pi K} \sum_{\alpha, \nu} \int_{0}^{L \Lambda} d x \int_{0}^{K / t} d \tau\left\{\quad\left[\left(\partial_{x} \varphi_{\alpha}\right)^{2}+\left(\partial_{\tau} \varphi_{\alpha}\right)^{2}\right] \delta_{\alpha \nu}-\frac{1}{2 K} \int_{0}^{K / t} d \tau^{\prime}\left[u^{2} \cos p\left(\varphi_{\alpha}(x, \tau)-\varphi_{\nu}\left(x, \tau^{\prime}\right)\right)\right.\right. \\
\left.\left.+\sigma \partial_{x} \varphi_{\alpha}(x, \tau) \partial_{x} \varphi_{\nu}\left(x, \tau^{\prime}\right)\right]\right\},
\end{gathered}
$$

with $\sigma=2 u^{2}\left(\rho_{0} \Lambda / \rho_{1} Q\right)^{2}$.

Note, that we introduced dimensionless spatial and imaginary time variables,

$$
\begin{gathered}
\Lambda x \rightarrow x, \\
\Lambda v \tau \rightarrow \tau,
\end{gathered}
$$

which will be used throughout the paper - beginning here. Furthermore all lengths (e.g. correlation lengths, $\lambda_{T}, L_{F L}, l_{i m p}$ and $L$ ), wave vectors (e.g. $k, k_{F}$ and
$Q)$ and Matsubara frequencies are dimensionless accordingly, from now on. Additionally we rescale the elastic constant

$$
\Lambda c \rightarrow c,
$$

for convenience to avoid the appearance of $\Lambda$.

Integrating over the high momentum modes of $\varphi(x, \tau)$ in a momentum shell of infinitesimal width $1 / b \leq|q| \leq 1$ 
but arbitrary frequencies and rescaling $x \rightarrow x^{\prime}=x / b$, $\tau \rightarrow \tau^{\prime}=\tau / b$, we obtain the following renormalization group flow equations (up to one loop):

$$
\begin{aligned}
\frac{d t}{d l} & =t \\
\frac{d K}{d l} & =-\frac{1}{2} p^{4} u^{2} K B_{0}\left(p^{2} K, \frac{K}{2 t}\right) \operatorname{coth} \frac{K}{2 t}, \\
\frac{d u^{2}}{d l} & =\left[3-\frac{p^{2} K}{2} \operatorname{coth} \frac{K}{2 t}\right] u^{2}, \\
\frac{d \sigma}{d l} & =\sigma
\end{aligned}
$$

where $l=\ln b$. For details on the RG calculation we relegate to Appendix $\mathrm{A}$ where we have written the $\mathrm{RG}$ flow also for dimensions $0<|d-1| \ll 1$.

For legibility we have introduced the following functions:

$$
\begin{aligned}
& B_{i}(\nu, y)=\int_{0}^{y} d \tau \int_{0}^{\infty} d x \frac{g_{i}(\tau, x)}{\Upsilon(\tau, x)} \frac{\cosh (y-\tau)}{\cosh y} \\
& \Upsilon(\tau, x)=\left[1+\left(\frac{y}{\pi}\right)^{2}\left(\cosh \frac{\pi x}{y}-\cos \frac{\pi \tau}{y}\right)\right]^{\nu / 4}
\end{aligned}
$$

with

$$
g_{0}(\tau, x)=\delta(x) \tau^{2},
$$

Note, that $B_{0}\left(p^{2} K, \frac{K}{2 t}\right) \rightarrow 0$ for $K \rightarrow 0$ (see Fig. 5 in Appendix A).

There is no renormalization of $t$ (i.e., of the elastic constant $c$ ) because of a statistical tilt symmetry ${ }^{25}$. Note that (18) is written in rescaled dimensionless parameters and the different renormalization of the kinetic and elastic term is reflected in the different renormalization of $v$ and $c$, i.e., $K$ and $t$, respectively.

From the flow equation for $u^{2}$ (19c) one sees directly that, depending on the sign of the prefactor, the behavior changes from increase for small $t$ and $K$ to decrease for high $K$ or $t$.

There is no first order RG correction to $\sigma$ and the change of $\sigma$ with length scale is simply given by rescaling, see (19d). The two-loop contribution to $\sigma$ is much more involved than the one-loop contributions for the other flow equations and gives no qualitatively different result for the flow of $\sigma$. As seen from (19d), the forward scattering amplitude always increases as $\sigma_{0} e^{l}$ on larger length scales and is therefore not well controlled in the RG sense. But, since the flow of $\sigma$ does not feed back into the other flow equations it has only minor relevance for our considerations. And indeed, we can get rid of the forward scattering term $f / \pi U(x) \frac{\partial \varphi}{\partial x}$ by introducing the field $\hat{\varphi}_{b}(x)$ by

$$
\hat{\varphi}(x)=\hat{\varphi}_{b}(x)-\varphi_{f}(x), \quad \varphi_{f}(x)=\int_{0}^{x} d y c(y),
$$

where $c(x) \equiv \frac{U(x) f}{\pi c \Lambda}$, with $\overline{c(x)}=0$ and $\overline{c(x) c\left(x^{\prime}\right)}=$ $\frac{\pi}{2} \sigma \delta\left(x-x^{\prime}\right)$. This can be easily verified by inserting this decomposition of $\hat{\varphi}(x)$ into the initial Hamiltonian (4) written in dimensionless units, and using (5) and the definition of $\sigma$ for deriving the averages of $c(x)$. Note, that $x$ is dimensionless.

TABLE I: Notation guide.

\begin{tabular}{ccc}
\hline \hline symbol here & G. \& S. ${ }^{a}$, Ref. [11] & Haldane, Ref. [31] \\
\hline$\varphi, \hat{j}$ & $\sqrt{2} \phi, \sqrt{2} / \pi \partial_{\tau} \phi$ & $\theta-\pi \rho_{0} x, \pi^{-1} \dot{\theta}$ \\
$\hat{P}$ & $\hbar \Pi / \sqrt{2}$ & $-\frac{\hbar}{\pi} \nabla \varphi$ \\
$K$ & $2 K_{\rho}$ & $\sqrt{v_{j} / v_{N}}$ \\
$v$ & $u_{\rho}$ & $\sqrt{v_{j} v_{N}}$ \\
$c$ & $\frac{\hbar u_{\rho}}{2 \pi K_{\rho}}$ & $\hbar v_{N} / \pi$ \\
$p$ & 1 & 2 \\
\hline \hline
\end{tabular}

${ }^{a}$ charge operators

\section{B. Zero temperature - a review}

The special case $t=0$ was previously considered, e.g., in 11] (for a better comparison see our notation guide given in table (1).

The flow equations for $K$ and $u$ at zero temperature read:

$$
\begin{aligned}
\frac{d K}{d l} & =-\frac{1}{2} p^{4} u^{2} K B_{0}\left(p^{2} K, \infty\right), \\
\frac{d u^{2}}{d l} & =\left[3-\frac{p^{2} K}{2}\right] u^{2},
\end{aligned}
$$

with

$$
B_{0}(\nu, \infty)=\int_{0}^{\infty} d \tau \tau^{2} e^{-\tau}\left[1+\tau^{2} / 2\right]^{-\nu / 4} .
$$

The corresponding flow equation for $K$ obtained in 11 . deviates slightly from (23a), which can be traced back to the different $\mathrm{RG}$ procedures. In [11] the authors performed the RG at strictly zero temperature and used a symmetric, circular shape of the "momentum-shell", i.e., treated the model as effectively isotropic in the $1+1$ dimensional space-time.

This procedure may be a good approximation at zero temperature, but if one considerers finite temperatures this does not hold anymore, since the extension in $\tau$ direction is now finite. As a result, there is a region $\pi / L<|k|<\pi / \lambda_{T}$ where fluctuations are mainly onedimensional and purely thermal. This region was disregarded in previous treatments. As we will see, fluctuations from this region have an important influence on the overall phase diagram.

The critical behavior is, however, the same: there is a Kosterlitz-Thouless (KT) transition at the phase boundary $K_{u}$ between a disorder dominated, pinned and a free, unpinned phase which terminates in the fixed point 
$K_{u}^{*}=6 / p^{2}$. One can derive an implicit equation for $K_{u}$ by combining (23a) and 23b to a differential equation

$$
\frac{d u^{2}}{d K}=\frac{1}{p^{2} \eta K}\left(K-K_{u}^{*}\right)
$$

which has the solution

$$
u^{2}(K)-u_{0}^{2}=\frac{K_{u}^{*}}{p^{2} \eta}\left(\frac{K-K_{0}}{K_{u}^{*}}-\ln \frac{K}{K_{0}}\right),
$$

where $u_{0}$ and $K_{0}$ denote the bare values of the disorder and quantum fluctuation, respectively, and $\eta \equiv$ $B_{0}\left(p^{2} K_{u}^{*}, \infty\right)$. Then, $K_{u}$ is implicitly given by

$$
u^{2}\left(K_{u}\right)=\frac{K_{u}^{*}}{p^{2} \eta}\left(\frac{K_{u}-K_{u}^{*}}{K_{u}^{*}}-\ln \frac{K_{u}}{K_{u}^{*}}\right),
$$

where the initial condition $u^{2}\left(K_{0}=K_{u}^{*}\right)=u_{0}^{2}=0$ is used. The KT-flow equations at $K_{u}^{*}$ can be recovered by defining

$$
\begin{aligned}
2 \gamma & \equiv \frac{p^{2} K}{2}-3, \\
2 \chi^{2} & \equiv \frac{3}{2} p^{4} \eta u^{2}
\end{aligned}
$$

with $|\gamma| \ll 1$. This yields

$$
\begin{gathered}
\frac{d \gamma}{d l}=-\chi^{2}, \\
\frac{d \chi^{2}}{d l}=-2 \gamma \chi^{2},
\end{gathered}
$$

which are exactly the flow equations obtained by Kosterlitz and Thouless 26 .

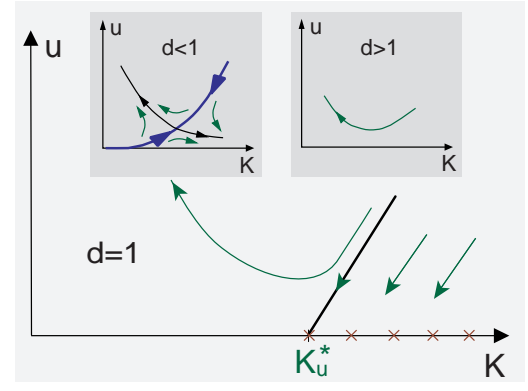

FIG. 1: Schematic zero temperature phase diagram in $d=1$ and close to $d=1$ dimensions (see text). $u$ and $K$ denote the strength of the disorder and quantum fluctuations, respectively.

Under the assumption, that a small deviation from the dimension $d=1$ changes only the naive scaling dimensions of the fields, our results can be extended also to $d=1+\epsilon$ dimensions (For details see Appendix B). The zero temperature phase diagram is modified and illustrated in Fig. 1] For $\epsilon<0$ the fixed point at $\left(K=K_{u}^{*}\right.$, $u=0$ ) is shifted to positive $u$-values (see left inset of
Fig. 11), whereas for $\epsilon>0, K$ and $u$ always flow to the strong pinning fixed point (at $K=0$ and $u \rightarrow \infty$; right inset), i.e., quantum fluctuations are too weak to renormalize the random potential to zero. The zero temperature transition disappears therefore for $d>1$, since the fixed point lies in the unphysical $u<0$ region of the $K-u$ parameter space. This can easily be verified by using the rescaling of $K$ given in eq. (A12C) of Appendix A which results in the flow equation (A16). In general this discussion applies to the localization transition as well as to the Mott transition (see discussion of the the lattice potential). Note, that the flow for $d \neq 1$ is qualitatively different from that discussed in Ref. 27].

If one includes the effect of Coulomb interaction in $d=1$ dimension, phase fluctuations of the free phase field increase only as $(\mathrm{T}=0)$

$$
\left\langle(\varphi(x, 0)-\varphi(0,0))^{2}\right\rangle \sim K \ln ^{1 / 2}|x| .
$$

As a result, phase fluctuations are too weak to suppress the disorder even for large values of $K$ and the system is always in the pinned phase. The phase diagram is therefore similar to that in $d>1$ dimensions.

In the pinned phase the parameters $K$ and $u$ flow into the classical, strong disorder region: $K \rightarrow 0, u \rightarrow \infty$.

Integration of the flow equations gives for small initial disorder and $K \ll K_{u}^{*}$ an effective correlation length or localization length

$$
\xi_{u} \approx L_{F L}^{\left(1-K / K_{u}^{*}\right)^{-1}}
$$

at which $u$ becomes of the order unity. This can be extracted from 23b neglecting the flow of $K$.

A better approximation of $\xi_{u}$ which takes also the flow of $K$ into account can be obtained by replacing $u^{2}$ in the flow equation for $K$ 23a by the expression given in (26). We still use the approximation, that $K$ deviates not much from the bare value $K_{0}$ which is the case, as long as $u_{0}^{2} l \ll 1$. Then, the solution for $K(l)$ is given by

$$
K(l) \approx K_{0}\left(1-\frac{p^{4}}{2} u_{0}^{2} \eta l\right),
$$

which yields a solution of (23b):

$$
\ln \frac{u^{2}(l)}{u_{0}^{2}} \approx\left(3-\frac{p^{2}}{2} K_{0}\right) l+\frac{p^{6}}{8} \eta K_{0} u_{0}^{2} l^{2} .
$$

With $u^{2}\left(\ln \left(\xi_{u}\right)\right) \approx 1$, the correlation length $\xi_{u}$ is defined by

$$
0=\ln u_{0}^{2}+\underbrace{\left(3-\frac{p^{2}}{2} K_{0}\right)}_{\equiv a} \ln \left(\xi_{u}\right)+\underbrace{\frac{p^{6}}{8} \eta K_{0} u_{0}^{2}}_{\equiv b}\left(\ln \left(\xi_{u}\right)\right)^{2}
$$

which yields

$$
\begin{aligned}
\ln \left(\xi_{u}\right) & =\frac{\sqrt{a^{2}-4 b \ln u_{0}^{2}}-a}{2 b} \\
& \approx-\frac{\ln u_{0}^{2}}{3-\frac{p^{2} K_{0}}{2}}-\frac{p^{6}}{8} \eta K_{0} u_{0}^{2} \frac{\left(\ln u_{0}^{2}\right)^{2}}{\left(3-\frac{p^{2} K_{0}}{2}\right)^{3}}
\end{aligned}
$$


where the first term of the r.h.s. gives the result (30).

Close to the transition line $\xi_{u}$ shows KT behavior. For $K \geq K_{u}, \xi_{u}$ diverges and $C(x, \tau) \sim K(l=\ln |z|) \ln |z|$ where $|z|=\sqrt{x^{2}+\tau^{2}}$ (cf. section [V).

\section{Strong pinning limit: Exact ground state}

For large values of $u$ our flow equations break down. Qualitatively the flow is towards large $u$ and small $K$. We can, however, find the asymptotic behavior in this phase by solving the initial model in the strong pinning limit exactly. To find this solution we will assume strong pinning centers and weak thermal fluctuations:

$$
U_{i m p} \rightarrow \infty \text { and } c / l_{i m p} \gg T \text {. }
$$

To treat this case we go back to the initial Hamiltonian (4) (with $W \equiv 0$ and the kinetic term also vanishes because of $K \rightarrow 0$ ). For strong disorder it is convenient to integrate out the phase field $\varphi(x)$ at all points which are not affected by the impurities. Then the effective Hamiltonian takes the form ${ }^{8}$

$$
\mathcal{H}_{\text {eff }}=\sum_{i=1}^{N}\left\{\frac{c}{2} \frac{\left(\varphi_{i+1}-\varphi_{i}\right)^{2}}{x_{i+1}-x_{i}}+U_{i} \rho\left(x_{i}\right)\right\}, \varphi_{i} \equiv \varphi\left(x_{i}\right) .
$$

Under condition (35), $\varphi_{i}$ only takes values obeying

$$
p\left(\varphi_{i}+Q x_{i}\right)=2 \pi n_{i}+\pi \text { with } n_{i} \in \mathbb{Z} \text { integer }
$$

which minimizes the backward scattering term. Defining $h_{i}$ and $\epsilon_{i}$ by

$$
n_{i+1}-n_{i} \equiv h_{i}+\left[\frac{p Q l_{i m p}}{2 \pi}\right], \quad x_{i+1}-x_{i} \equiv l_{i m p}+\epsilon_{i}
$$

with $0 \leq x_{1} \leq x_{2} \leq \ldots \leq x_{N+1} \leq L$, the effective Hamiltonian can be rewritten as

$$
\mathcal{H}_{\text {eff }}=\frac{c}{2 p^{2}} \sum_{i} \frac{(2 \pi)^{2}\left(h_{i}-\frac{p Q \epsilon_{i}}{2 \pi}-\gamma\right)^{2}}{l_{i m p}+\epsilon_{i}} .
$$

Here $[x]$ denotes the closest integer to $x$ (Gaussian brackets):

$$
\left.[x]=m \text { for } x \in] m-\frac{1}{2}, m+\frac{1}{2}\right], m \in \mathbb{Z}
$$

and

$$
\gamma \equiv \frac{p Q l_{i m p}}{2 \pi}-\left[\frac{p Q l_{i m p}}{2 \pi}\right],
$$

such that $|\gamma| \leq \frac{1}{2}$.

Because thermal fluctuations are small compared to the elastic energy, see (35), $\left(h_{i}-\frac{p Q \epsilon_{i}}{2 \pi}-\gamma\right)$ takes on its minimal value, which is given by

$$
h_{i}^{0}=\left[\frac{p Q \epsilon_{i}}{2 \pi}+\gamma\right] \text {. }
$$

which defines the exact ground state of the classical model: If we use (38) one finds for the optimal value of the $n_{i}$ 's

$$
n_{i+1}^{0}=n_{i}^{0}+\left[\frac{p Q}{2 \pi}\left(\epsilon_{i}+l_{i m p}\right)\right]
$$

which leads, using (37), to the exact classical ground state

$$
\varphi_{i}^{0}=\frac{1}{p}\left(2 \pi\left\{n_{1}^{0}+\sum_{j<i}\left[\frac{p Q}{2 \pi}\left(\epsilon_{j}+l_{i m p}\right)\right]\right\}+\pi\right)-Q x_{i},
$$

where $n_{1}^{0}$ has an arbitrary integer value.

\section{Finite temperature and crossover diagram}

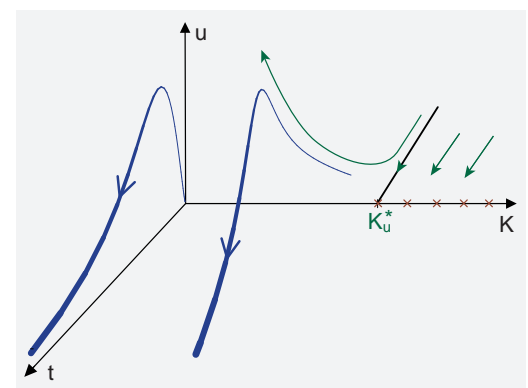

FIG. 2: Typical flow diagram for $w=0$ in the three dimensional parameter space of $K, u$ and $t$, proportional to the strength of quantum, disorder and thermal fluctuations, respectively.

At finite temperatures thermal fluctuations wipe out the random potential which lead to the pinning of the CDW at $t=0$ and $K<K_{u}$. Thus there is no phase transition any more, in agreement with the Landau theorem. The system is always in its delocalized phase even if the disorder may still play a significant role on intermediate length scales.

In the special case $K \rightarrow 0$ the flow equation (19c) reduces to $\frac{d u^{2}}{d l}=\left[3-p^{2} t\right] u^{2}$ with solution

$$
u^{2}(l)=u_{0}^{2} e^{3 l-p^{2} t_{0}\left(e^{l}-1\right)} .
$$

If we write $t=t_{0} e^{l}$ we may express $l$ by $t$ and hence we may write $u^{2}$ as $t$-dependent function:

$$
u^{2}(t)=u_{0}^{2}\left(t / t_{0}\right)^{3} e^{-p^{2}\left(t-t_{0}\right)},
$$

which is plotted in Fig. 2 in the $t$ - $u$ plane.

One sees that the flow of the disorder has a maximum at $t=3 / p^{2}$ or $l=\ln \left(3 /\left(p^{2} t_{0}\right)\right)$, if $t_{0}<3 / p^{2}$. For finite $K$, the $\mathrm{RG}$ flow of $u$ in the region $K<K_{u}$ first increases and then decreases. The region of increase in the $K-t$ plane is implicitly defined by $\mathcal{M}_{u} \equiv$ 
$\left\{(K, t) \mid K_{u}^{*} \geq K \operatorname{coth} \frac{K}{2 t} \geq 0\right\}$, i.e., the positions of the maxima of $u^{2}[K, t]$ are located on the boundary of $\mathcal{M}_{u}$ defined by $K_{u}^{*}=K \operatorname{coth} \frac{K}{2 t}$.

The correlation length $\xi$ can be found approximately by integrating the flow equations until the maximum of $u(l)$ and $t(l) /(1+K(l))$ is of the order one (see discussion in section IV. This can be done in full generality only numerically (see Fig. [3).

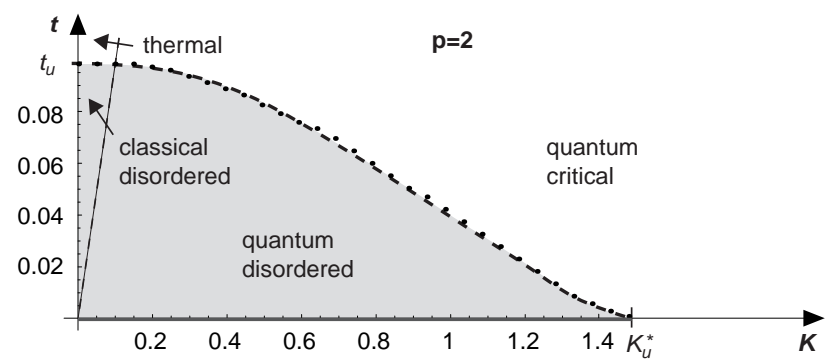

FIG. 3: The low temperature crossover diagram of a onedimensional CDW. $t$ and $K$ are proportional to the temperature and the strength of quantum fluctuations, respectively. The amount of disorder corresponds to a reduced temperature $t_{u} \approx 0.1$. In the classical and quantum disordered region, respectively, essentially the $t=0$ behavior is seen. The straight dashed line separating them corresponds to $\lambda_{T} \approx 1$, i.e., $K \approx t$. In the quantum critical region the correlation length is given by $\lambda_{T}$. Pinning (localization) occurs only for $t=0, K<K_{u}^{*}$.

It is however possible to discuss several special cases analytically. The zero temperature correlation length can still be observed as long as this is smaller than the thermal de Brogle wave length $\lambda_{T}$ which can be rewritten for $K$ not too close to $K_{u}^{*}$ as $t \lesssim t_{K} \approx K t_{u}^{\left(1-K / K_{u}\right)^{-1}}$ with $t_{u} \approx L_{F L}^{-1}$, where we defined $t_{K}$ via $\xi_{u} \equiv \frac{K}{t_{K}}$, analogously to the definition of $\lambda_{T}$, and used (30). We call this domain the quantum disordered region.

For $K \geq K_{u}$ the correlation length $\xi$ is given by $\lambda_{T}$ which is larger than given by purely thermal fluctuations. For scales smaller than $\lambda_{T}$, the phase correlation function still increases as $\sim \ln |z|$ with a continuously varying coefficient $K_{\text {eff }}\left(u_{0}\right)$, as will be discussed in detail in the next section. In this sense one observes quantum critical behavior in that region, despite of the fact, that the correlation length is now finite for all values of $K[12]$.

In the classical disordered region $t_{K}<t<t_{u}$ the correlation length is roughly given by $L_{F L}$ as follows from previous studies.8 or by solving $u^{2}(\ln (\xi)) \simeq 1$ using (44) for small $t_{0}$ yielding $\xi \approx u_{0}^{-2 / 3} e^{-p^{2} t_{0}} \approx u_{0}^{-2 / 3}=$ $L_{F L}\left(\pi p^{4}\right)^{1 / 3}$. Note, that $t_{K} \approx K$ for small $K$.

In the remaining region $t \gtrsim t_{u}$, the thermal region, we apply the mapping onto the Burgers equation (see section [V]. In this case the RG-procedure applied to this equation becomes trivial since there is only a contribution from a single momentum shell and one finds for the correlation length $\xi^{-1} \approx \frac{\pi}{2} f(T) t\left[1+1 / 2\left[t_{u} /\left(\pi p^{2} t\right)\right]^{3}\right] \Lambda$.

The phase diagram depicted in Fig. B is the result of the numerical integration of our flow equations and shows indeed the various crossovers discussed before.

In the high temperature region $(t \gg K)$ the flow equations can be solved explicitly. For $u^{2}(l)$ we get the same result as given in (44) and the flow equation for $K$ reduces to

$$
\frac{d K}{d l}=-\frac{p^{4}}{2} u^{2} \frac{K^{4}}{(2 t)^{3}}
$$

where we used $B_{0}\left(p^{2} K, \frac{K}{2 t} \rightarrow 0\right)=(K / 2 t)^{4}$. The solution of this equation is given by

$$
K(l)=\left[K_{0}^{-3}+\frac{3 p^{4} u_{0}^{2}}{16 t_{0}^{3}} e^{p^{2} t_{0}} \operatorname{Ei}\left(p^{2} t_{0}, p^{2} t_{0} e^{l}\right)\right]^{-1 / 3},
$$

with the incomplete exponential integral function $\operatorname{Ei}(a, b)$ defined by

$$
\operatorname{Ei}(a, b) \equiv \int_{a}^{b} d t e^{-t} / t .
$$

One observes that $K(l)$ saturates very quickly to the value $K(\infty)<K_{0}$.

\section{CORRELATION FUNCTIONS}

In this section we discuss the density-density and the phase correlation functions in more detail and summarize all correlation lengths in the various regimes - partly already used in the last two sections.

The (full) density-density correlation function is defined as

$$
S(x, \tau) \equiv\langle\rho(x, \tau) \rho(0,0)\rangle,
$$

where $\rho(x, \tau)$ is given in (11). In the following we restrict our considerations to the (charge) density wave order part of $S$, which is the term proportional to $\rho_{1}^{2}$, i.e.

$$
S_{1}(x, \tau)=\rho_{1}^{2}\langle\cos p(\varphi(x, \tau)+Q x) \cos p \varphi(0,0)\rangle,
$$

which defines the type of order of the density wave: If it decays algebraically we have quasi long-range order (QLRO), an exponential decay over a correlation length $\xi$ corresponds to short-range order (SRO). The omitted parts of $S$ decay faster than $S_{1}[28]$.

$S_{1}$ can be rewritten as

$$
\begin{aligned}
S_{1}(x, \tau)=\frac{\rho_{1}^{2}}{4}(\quad & e^{\imath p Q x}\left\langle e^{\imath p(\varphi(x, \tau)-\varphi(0,0))}\right\rangle+ \\
& \left.e^{-\imath p Q x}\left\langle e^{-\imath p(\varphi(x, \tau)-\varphi(0,0))}\right\rangle\right),
\end{aligned}
$$

and under the assumption that we can use a gaussian approximation for the averages, we obtain

$$
S_{1}(x, \tau) \simeq \rho_{1}^{2} \cos (p Q x) e^{-\frac{p^{2}}{2}\left\langle(\varphi(x, \tau)-\varphi(0,0))^{2}\right\rangle} .
$$


From now on we focus on the phase correlation function

$$
C(x, \tau) \equiv\left\langle(\varphi(x, \tau)-\varphi(0,0))^{2}\right\rangle,
$$

and discuss it in various limits. Combining (51) and (52) we can extract a correlation length from the relation

$$
\xi^{-1}=\lim _{x \rightarrow \infty} \frac{p^{2}}{2 x} C(x, 0)
$$

\section{A. Disorder-free case}

We start with the most simple case $u=0$. Then, the correlation function in dimensionless units follows directly from the action $\mathcal{S}_{0}$ written in momentum space:

$$
C_{0}(x, \tau)=\frac{2 \pi t}{L} \sum_{k, n} \frac{1-e^{\imath\left(k x+\omega_{n} \tau\right)}}{\omega_{n}^{2}+k^{2}},
$$

with Matsubara frequencies $\omega_{n}=2 \pi n / \lambda_{T}$ and momenta $\left.k=k_{m}=2 \pi m / L\right)$.

The sums over $n$ and $k$ (i.e., $m$ ) can be performed approximately for sufficiently large $x$ and $\tau$ and one obtains 29

$$
\begin{aligned}
C_{0}(x, \tau) & \simeq \frac{K}{2} \ln (1+ \\
& \left.\left(\frac{\lambda_{T}}{2 \pi}\right)^{2}\left[\cosh \left(\frac{2 \pi x}{\lambda_{T}}\right)-\cos \left(\frac{2 \pi \tau}{\lambda_{T}}\right)\right]\right) .
\end{aligned}
$$

The behavior of this function is considered in the following cases:

(i) At zero temperature $\left(\lambda_{T} \rightarrow \infty\right)$ [55) reduces to

$$
C_{0}(x, \tau) \simeq \frac{K}{2} \ln \left(\frac{1}{2}\left[x^{2}+\tau^{2}\right]+1\right),
$$

i.e., the correlation function has a logarithmic dependency on $x$ and $\tau$ and leads to an algebraic decay of $S_{1}$, i.e., the system shows QLRO.

(ii) At finite temperatures we can distinguish between length scales smaller and larger than $\lambda_{T}$.

In the first case $x \ll \lambda_{T}$ and $\tau \ll \lambda_{T}$ the cosh and cos term can be expanded to second order in the arguments and one gets the same logarithmic function as in the zero temperature case. In the opposite case $x \gg \lambda_{T}$, which is the usual case at high temperatures, the cosh term can be approximated by the exponential function and one finds a linear dependency on $x$ :

$$
C_{0}(x) \approx \pi t x=T x / c \quad \text { i.e. } \quad \xi=\frac{2}{p^{2} \pi t} \equiv \xi_{T},
$$

i.e., $S_{1}$ decays exponentially (SRO) over a characteristic length $\xi \sim t^{-1}$. The same result is obtained for the limit $K \rightarrow 0$ at a fixed, finite temperature.
Note, that with this result we have neglected the algebraic decay for small $x<\lambda_{T}$. Therefore a better interpolation formula for the correlation length is $\xi \approx \frac{2}{p^{2}}\left(\xi_{T}+\lambda_{T}\right)$, which takes the slow decay for small $x$ into account. In terms of the length-scale dependent $t(l)$ this rewrites to

$$
t(l=\ln (\xi))=K+1,
$$

i.e., the correlation length is reached, if $t(l) /(1+K)$ is of order one.

The change from QLRO on small length scales $x<\xi$ to SRO on large length scales becomes clear if one considers the cylindric topology of the system in space-time at finite temperatures: As soon as one reaches length scales of the order of the perimeter of the cylinder, which is $\lambda_{T}$, starting from small scales, the system changes from two-dimensional to effectively one-dimensional behavior.

\section{B. Finite disorder}

If $u$ is finite the action of the system has a forward and a backward scattering part. With the decomposition (22), the phase correlation function divides into two parts:

$$
C(x, \tau)=C_{b}(x, \tau)+C_{f}(x)
$$

and has therefore always a contribution $C_{f}(x) \sim|x| / \xi_{f}$ with $\xi_{f}^{-1} \sim \sigma(l=\ln |x|)$, i.e., the density wave order has always an exponentially decaying contribution and we can define

$$
S_{1}(x, \tau) \equiv f_{\rho}(x) e^{-\frac{p^{2}}{2} C_{b}(x, \tau)},
$$

with $f_{\rho}(x)=\rho_{1}^{2} \cos (p Q x) e^{-\frac{p^{2} \pi}{4}|x| / \xi_{f}}$. However, since $C_{f}(x)$ is not $\tau$-dependent, it will not influence the dynamical properties of the system. Therefore all further remarks about phase correlations refer to $C_{b}(x, \tau)$ and consequently we will drop the subscript $b$ in the following. Again we examine the $T=0$ and finite temperature cases:

(i) At zero temperature we have to distinguish between three $K$-regimes: For $K>K_{u}$ the disorder becomes irrelevant under the RG flow and we can use the zero temperature, disorder free result for the correlation function with the pre-factor $K$ replaced by an effective quantity $K_{\text {eff }}(l=\ln z)$ on a length scale $z=\sqrt{x^{2}+\tau^{2}}$, defined by the flow equation for $K$. This effective $K$ saturates on large scales at a value $K_{\text {eff }}\left(u_{0}\right)$, which may be seen in Fig. 2] Therefore we have QLRO in this $K$ region.

For $0<K<K_{u}$ we integrate the flow of $u$ until it reaches a value of order one, starting at small $u_{0}$, which defines the localization length $\xi_{u}$ (see section the correlation function behaves like $C(x, \tau) \sim|x| / \xi_{u}$, i.e., we have an additional (to $C_{f}$ ) exponentially decaying contribution to $S_{1}$. 


\section{Strong disorder}

In the last region $K=0$ we come back to the strong pinning case, discussed in section $\amalg$ IIC before and calculate the pair correlation function exactly. Taking into account that the $h_{i}$ 's are independent on different lattice sites, i.e., $\overline{h_{i} h_{j}} \propto \delta_{i j}$, the (discrete) phase correlation function is given by

$$
\begin{aligned}
\overline{\left\langle\left(\varphi_{n+1}-\varphi_{1}\right)^{2}\right\rangle} & =\frac{4 \pi^{2}}{p^{2}} \overline{\left\langle\left(h_{i}-\frac{p Q \epsilon_{i}}{2 \pi}-\gamma\right)^{2}\right\rangle} \cdot n \\
& =\frac{4 \pi^{2}}{p^{2}} \overline{\left(\frac{p Q \epsilon_{i}}{2 \pi}+\gamma-\left[\frac{p Q \epsilon_{i}}{2 \pi}+\gamma\right]\right)^{2}} n,
\end{aligned}
$$

where we used (42) for the second equality. For evaluating the disorder average in this expression, one has to take into account the order statistics of the impurity distances $\epsilon_{i}$. In the thermodynamic limit the probability density function for the $\epsilon_{i}$ 's can be rewritten as

$$
p\left(\epsilon_{i}\right) \approx \frac{l_{i m p}^{-1}}{e} e^{-l_{i m p}^{-1} \epsilon_{i}}, \quad-l_{i m p} \leq \epsilon_{i}<\infty .
$$

Then, the correlation function can be explicitly written as

$$
\overline{\left\langle\left(\varphi_{n+1}-\varphi_{1}\right)^{2}\right\rangle}=\frac{4 \pi^{2}}{p^{2}} \int_{0}^{\infty} d x e^{-x}\left(\frac{x}{2 \alpha}-\left[\frac{x}{2 \alpha}\right]\right)^{2} n,
$$

where we introduced the parameter $\alpha \equiv \frac{\pi}{p Q l_{i m p}}$ and substituted $x=l_{i m p}^{-1} \epsilon_{i}+1$. This integral can be evaluated exactly, which leads to the following exact expression for the pair correlation function at zero temperature, written in a continuum version:

$$
C(x, \tau)=\frac{2 \pi}{p \alpha}\left(1-\frac{\alpha}{\sinh \alpha}\right)|Q x| \equiv \frac{2 x}{p^{2} \xi}, \quad \alpha=\frac{\pi}{p Q l_{i m p}} .
$$

A more detailed derivation of this result is given in Appendix B

Finally we want give an interpolating expression for $C(x, \tau)$ from $T=0$ to high temperatures $T \gg c /\left(l_{\text {imp }} p^{2}\right)$ starting with the result (63). In the latter case we may neglect the discreteness of $h_{i}$ and hence

$$
\begin{aligned}
& \overline{\left\langle\left(\varphi_{n+1}-\varphi_{1}\right)^{2}\right\rangle} \\
& \approx \frac{4 \pi^{2}}{p^{2} l_{i m p}} \overline{\left(-\frac{\partial}{\partial \lambda_{1}} \ln \left(\int d h e^{-\sum_{i} \lambda_{i} h^{2}}\right)\right)}|x| \\
& =\frac{T}{c}|x|=\pi t|x|,
\end{aligned}
$$

with $\lambda_{i}=\frac{2 \pi^{2} c}{T p^{2}\left(l_{i m p}+\epsilon_{i}\right)}$.

A plausible interpolation formula is then given by

$$
\overline{\left\langle(\varphi(x)-\varphi(0))^{2}\right\rangle} \approx\left(2 Q^{2} l_{i m p}\left(1-\frac{\alpha}{\sinh (\alpha)}\right)+\frac{T}{c}\right)|x|,
$$

and for $l_{i m p} \gg Q^{-1}$, i.e., $\alpha \ll 1$ :

$$
\overline{\left\langle(\varphi(x)-\varphi(0))^{2}\right\rangle} \approx\left(\frac{\pi^{2}}{3 p^{2}} l_{i m p}^{-1}-\frac{7 \pi^{4}}{180 p^{4}} \frac{l_{i m p}^{-3}}{Q^{2}}+\frac{T}{c}\right)|x| .
$$

and hence the correlation length acquires the form

$$
\xi_{s p}^{-1} \approx p^{2} Q^{2} l_{i m p}\left(1-\frac{\alpha}{\sinh (\alpha)}\right)+\xi_{T}^{-1} .
$$

Note that $l_{i m p} Q \geq 1$, i.e., $\alpha \leq \pi / p$ and $\xi_{T} \gg l_{i m p}$. An approximate crossover to the weak pinning limit follows by choosing $l_{i m p} \approx L_{F L}$.

(ii) At finite temperatures the parameter $K$ saturates at an effective value $K_{\text {eff }}\left(u_{0}\right)$ on large length scale. Therefore the correlation function for small disorder is given by (55) with $K$ replaced by $K(l=\ln z)$.

In the region $\mathcal{M}_{u}$ of the $K$ - $t$ plane (see section IIID), $u$ still increases and we can find an effective correlation length by comparing the length scales on which $u(l)$ or $t(l) /(1+K(l)$ become of order one. Then, the correlation length is the smaller length of these two.

\section{Burgers equation}

For $K=0$, high temperatures but weak disorder we adopt an alternative method by mapping the (classical) one-dimensional problem onto the Burgers equation with noise $\mathrm{B}^{30}$. With this approach one can derive an effective correlation length given by

$$
\xi_{B}^{-1} \approx \xi_{T}^{-1}\left(1+\frac{1}{2}\left(\frac{\xi_{T}}{2 L_{F L}}\right)^{3}\right)
$$

where $\xi_{T} \ll L_{F L}$, which changes the prefactor of the free correlation function at high temperatures (57). The full calculation of this result can be found in Appendix C

TABLE II: Overview of the dimensionless correlation lengths.

\begin{tabular}{clc}
\hline \hline length & description & eq. \\
\hline$\xi_{B}$ & weak pinning/high temp. length & \\
$\xi_{f}$ & forward scattering length & \\
$\xi_{s p}$ & strong pinning length & high temp./disorder free length \\
$\xi_{T}$ & disorder localization length & \\
$\xi_{u}$ & lattice pot. correlation length & sec. $\mathbf{D 1}$ \\
$\xi_{w}$ &
\end{tabular}

\section{SUPERFLUIDS}

Next we consider the application of the results obtained so far to a one-dimensional Bose fluid. Its density 
operator is given by eq. (1) if we identify $Q f / \pi=\rho_{0}=\rho_{1}$ $(p=2)$ :

$$
\rho_{S F}=\frac{f}{\pi} \partial_{x} \varphi+\rho_{0}(1+\cos (2(\varphi+Q x)))+\ldots
$$

$\partial_{x} \varphi$ is conjugate to the phase $\theta$ of the Bose field operator $^{31}$. Keeping our definitions of $K, t$ and $u, v$ denotes now the phase velocity of the sound waves with $v=\sqrt{\kappa /\left(\rho_{0} m\right)}$ and the elastic constant is $c=\kappa /\left(\pi \rho_{0}\right)^{2}$, where $\kappa$ is the compressibility per unit length (see also table I).
With the replacements

$$
\begin{aligned}
K & \rightarrow K^{-1} \\
t & \rightarrow t / K^{2} \\
p & =2,
\end{aligned}
$$

(18) describes the action of the 1D-superfluid in a random potential. The correlation functions for the superfluid can be obtained correspondingly from this replacements. To avoid confusions we write down the full action in this case explicitly:

$$
\begin{aligned}
& \frac{\mathcal{S}_{S F}}{\hbar}=\frac{K}{2 \pi} \sum_{\alpha, \beta} \int_{0}^{L} d x \int_{0}^{K / t} d \tau \\
& \left\{\left[\left(\partial_{x} \varphi_{\alpha}\right)^{2}+\left(\partial_{\tau} \varphi_{\alpha}\right)^{2}\right] \delta_{\alpha \beta}-\frac{K}{2} \int_{0}^{K / t} d \tau^{\prime}\left[u^{2} \cos 2\left(\varphi_{\alpha}(x, \tau)-\varphi_{\beta}\left(x, \tau^{\prime}\right)\right)+\sigma \partial_{x} \varphi_{\alpha}(x, \tau) \partial_{x} \varphi_{\beta}\left(x, \tau^{\prime}\right)\right]\right\} .
\end{aligned}
$$

Hence the RG-equations follow from (19a to 19d with the above given replacements:

$$
\begin{aligned}
\frac{d t}{d l} & =\left[1+\frac{16 u^{2}}{K^{2}} B_{0}\left(4 / K, \frac{K}{2 t}\right) \operatorname{coth} \frac{K}{2 t}\right] t, \\
\frac{d K}{d l} & =\frac{8 u^{2}}{K} B_{0}\left(4 / K, \frac{K}{2 t}\right) \operatorname{coth} \frac{K}{2 t}, \\
\frac{d u^{2}}{d l} & =\left[3-\frac{2}{K} \operatorname{coth} \frac{K}{2 t}\right] u^{2}, \\
\frac{d \sigma}{d l} & =\sigma .
\end{aligned}
$$

I.e., the transition between the superfluid and the localized phase occurs at $K_{u}^{*}=2 / 3$ 11]. Thermal fluctuations again suppress the disorder and destroy the the superfluid localization transition in 1D.

\section{LATTICE POTENTIAL}

If the wave length $\lambda$ of the CDW modulation is commensurate with the period $a(=\pi$, due to dimensionless units) of the underlying lattice such that $n \lambda=q a$ with integers $n$ and $q$, the Umklapp term $-2 \pi(w / K) \cos (q \varphi)$ appears in the Hamiltonian ${ }^{1}$. Therefore we switch on the lattice potential $w \neq 0$ now. In this section we consider the case $u=0$ which leads to the Sine-Gordon type model:

$\frac{\mathcal{S}_{L P}}{\hbar}=\int_{0}^{L} d x \int_{0}^{K / t} d \tau \frac{1}{2 \pi K}\left\{\left(\partial_{x} \varphi\right)^{2}+\left(\partial_{\tau} \varphi\right)^{2}\right\}-\frac{w}{K} \cos (q \varphi)$
The model has $q$ degenerate classical ground states given by $\varphi_{m}=2 \pi m / q$ with $m=0, \ldots, q-1$. Performing a calculation analogous to the one above (but with $u=0$ ) the RG-flow equations read

$$
\begin{aligned}
\frac{d K}{d l} & =\frac{\pi}{2} q^{4} w^{2} B_{2}\left(q^{2} K, \frac{K}{2 t}\right) \operatorname{coth} \frac{K}{2 t} \\
\frac{d t}{d l} & =\left[1+\frac{\pi}{2} q^{4} w^{2} B_{1}\left(q^{2} K, \frac{K}{2 t}\right) \operatorname{coth} \frac{K}{2 t}\right] t, \\
\frac{d w}{d l} & =\left[2-\frac{q^{2}}{4} K \operatorname{coth} \frac{K}{2 t}\right] w
\end{aligned}
$$

where $B_{1,2}$ are given in (20) with

$$
\begin{aligned}
& g_{1}=2 x^{2} \cos x, \\
& g_{2}=\left(x^{2}+\tau^{2}\right) \cos x .
\end{aligned}
$$

Plots of the functions $B_{1}$ and $B_{2}$ can be found at the end of Appendix $\mathrm{A}$

At zero temperature (72a) and (72c) reduce to

$$
\begin{aligned}
\frac{d K}{d l} & =\frac{\pi}{2} q^{4} w^{2} B_{2}\left(q^{2} K, \infty\right), \\
\frac{d w}{d l} & =\left[2-\frac{q^{2}}{4} K\right] w,
\end{aligned}
$$

and we find, that for $u=0$ the lattice potential becomes relevant (i.e., $w$ grows) for $K<K_{w}$, where $K_{w}$ is implicitly defined by

$$
w^{2}\left(K_{w}\right)=\frac{K_{w}^{* 2}}{2 \pi q^{2} \tilde{\eta}}\left(\frac{K_{w}}{K_{w}^{*}}-1\right)^{2},
$$


which follows from

$$
\frac{d w}{d K}=-\frac{4}{q^{4} \pi \tilde{\eta} w}\left(1-\frac{K}{K_{w}^{*}}\right),
$$

where we used (73a) and (73b) and the initial condition $w\left(K_{w}^{*} \equiv 8 / q^{2}\right)=w_{0}=0 ; \tilde{\eta}=-B_{2}\left(q^{2} K_{w}^{*}, \infty\right)(\approx 0.4$, for $q=1)$.

In this region the periodic potential stabilizes true long-range order of the CDW: the phase is everywhere close to one of the $q$ classical ground states $\varphi_{m}$. The depinning transition from the lattice for $K \rightarrow K_{w}-0$ is again of KT type. The correlation length in the low- $K$ ordered phase $\xi_{w}$ is defined by $w\left(\ln \xi_{w}\right) \approx 1$ and diverges at $K_{w}-0$ [14]. This can be seen by considerations analogous to the disordered case. Defining

$$
\begin{aligned}
\gamma & =2 \frac{K}{K_{w}^{*}}-2, \\
\chi^{2} & =\frac{\pi}{8} q^{6} \tilde{\eta} w^{2}
\end{aligned}
$$

(note that $\tilde{\eta}>0$ ) leads for $|\gamma| \ll 1$, i.e., close to $K_{w}^{*}$, to the KT equations (28b) and (28a).

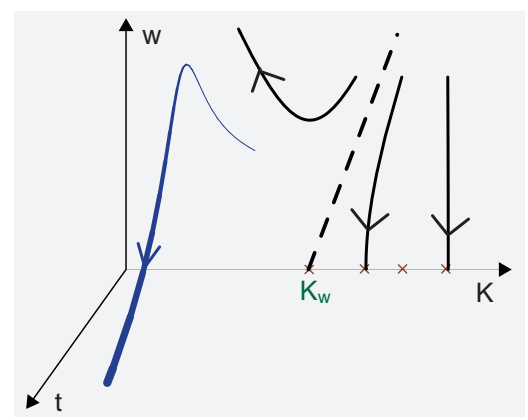

FIG. 4: Typical flow diagram for the disorder free model in the three dimensional parameter space of $K, w$ and $t$. $w$ denotes the strength of the commensurate lattice potential

At finite temperatures we find a similar scenario as in the case where we considered the influence of the disorder: $w$ first increases in a $K-t$ region given by $\mathcal{M}_{w} \equiv\left\{(K, t) \mid K_{w}^{*} \geq K \operatorname{coth} \frac{K}{2 t} \geq 0\right\}$, i.e., when the r.h.s. of $\sqrt{72 \mathrm{C}}$ is positive, but then decreases and flows into the region of large $t$ and small $w$. Thus the periodic potential becomes irrelevant at finite temperatures. This can be understood as follows: at finite $t$ the 1D quantum sine-Gordon model can be mapped on the Coulomb gas model on a torus of perimeter $\lambda_{T}$ since periodic boundary conditions apply now in the $\tau$-direction. Whereas the entropy of two opposite charges increases for separation $L \gg \lambda_{T}$ as $\ln \left(L \lambda_{T}\right)$, their action increases linearly with $L$. Thus, the charges remain bound. The onedimensional Coulomb gas has indeed only an insulating phase 32 .

\section{CONCLUSION}

To conclude we have shown that in one-dimensional charge and spin density waves, Luttinger liquids and suprafluids, quantum phase transitions between a disordered (or locked-in) phase and an asymptotically free phase at zero temperature are destroyed by thermal fluctuations leaving behind a rich crossover behavior. This was demonstrated by using a full finite temperature renormalization group (RG) calculation. The crossover regions were characterized by the behavior of the phase pair correlation functions. For vanishing quantum fluctuations our calculation was amended by an exact solution in the case of strong disorder and by a mapping onto the Burgers equation with noise in the case of weak disorder, respectively. Both methods gave an exponential decay of density correlations.

We have also briefly discussed, that the Coulomb interaction may destroy the unpinning (localization) transition.

The finite temperature calculation used in the present paper is also suited for treating the low frequency low temperature behavior of dynamical properties which may depend crucially on the ratio $\omega / T$. This as well as the discussion of the influence of quantum phase slips $\frac{16}{6}$ will be postponed to a forthcoming publication.

The combined effect of disorder and the lattice potential on the zero temperature phase diagram is still controversely discussed ${ }^{33.34}$ and cannot be explained by the RG-results presented in this paper, since both pertubations become relevant for small $K$. However, this problem is beyond the scope of the present work and may be discussed in a future publication.

\section{ACKNOWLEDGEMENT}

The authors thank A. Altland, S. Brasovskii, T. Emig, S. Korshunov, B. Rosenow, S. Scheidl and V. Vinokur for useful discussions. They acknowledge financial support by Deutsche Forschungsgemeinschaft through Sonderforschungsbereich 608. AG further acknowledges financial support by Deutscher Akademischer Austauschdienst.

\section{APPENDIX A: RENORMALIZATION OF THE DISORDER}

We present a short overview of the used finite temperature anisotropic renormalization group method in the case of the replicated disorder term. Starting point is the action (18).

$\begin{array}{ccc}\text { The phase field } & \varphi(x, \tau) & = \\ \frac{t}{K L \Lambda} \sum_{\omega_{n}} \sum_{|k| \leq 1} e^{\imath\left(\omega_{n} \tau+k x\right)} \varphi_{k, \omega_{n}} & \text { with the Matsub- }\end{array}$ $\frac{l}{K L \Lambda} \sum_{\omega_{n}} \sum_{|k| \leq 1} e^{\imath\left(\omega_{n} \tau+k x\right)} \varphi_{k, \omega_{n}}$ with the Matsub-
ara frequencies $\omega_{n}=2 \pi n t / K$ and $k=2 \pi m /(L \Lambda)$ (note that rescaled coordinates are used) is split in a slow $\left(|k|<b^{-1}\right)$ and a fast mode part $\left(b^{-1} \leq|k| \leq 1\right)$, where 
$b=e^{-d l}$ is a rescaling parameter of order 1 . Notice, that the $\varphi_{\lessgtr}$ still have all Matsubara Fourier components.

In order to find the RG-corrections of the other parameters in the model, we follow Wilson ${ }^{35}$ and expand $\left\langle\left\langle e^{-\mathcal{S}_{u}^{(n)} / \hbar}-1\right\rangle\right\rangle_{0,>}$ in small $(u / K)^{2}$, with

$\frac{\mathcal{S}_{u}^{(n)}}{\hbar}=-\frac{u^{2}}{4 \pi K^{2}} \sum_{\alpha, \beta} \iint d \tau d \tau^{\prime} \int d x R\left[\varphi_{\alpha}(x, \tau)-\varphi_{\beta}\left(x, \tau^{\prime}\right)\right]$

where $R[f] \equiv \cos (p f) .\langle\langle\ldots\rangle\rangle_{0,>}$ denotes the cumulative or connected average over the fast modes in the "momentum stripes" with the free gaussian model. The correction in first order is given by

$$
\frac{\mathcal{S}_{u, 1}^{(n)}}{\hbar}=\left\langle\left\langle\frac{\mathcal{S}_{u}^{(n)}}{\hbar}\right\rangle\right\rangle_{0,>}
$$

For calculating the cumulative average of the functional $R, R$ is expanded in small $\Delta \varphi_{>} \equiv \varphi_{\alpha,>}(x, \tau)-$ $\varphi_{\beta,>}\left(x, \tau^{\prime}\right)$, e.g.

$$
\langle\langle R[\Delta \varphi]\rangle\rangle_{0,>}=-p^{2}\left(R\left[\Delta \varphi_{<}\right]\left\langle\varphi_{\alpha,>}^{2}\right\rangle_{0,>}-R\left[\Delta \varphi_{<}\right]\left\langle\varphi_{\alpha,>}(x, \tau) \varphi_{\beta,>}\left(x, \tau^{\prime}\right)\right\rangle_{0,>}\right)+\mathcal{O}\left(\Delta \varphi_{>}^{4}\right) .
$$

The first term in A3 gives a correction to the disorder parameter $u$ and the second term a correction to $K$. The free thermal average over the fast modes can be evaluated with the free propagator $\left(k^{2}+\omega_{n}^{2}\right)^{-1}$ and using the formula

$$
\sum_{n=-\infty}^{\infty} \frac{\cos (n x)}{n^{2}+a^{2}}=\frac{\pi}{|a|} \frac{\cosh ((\pi-x) a)}{\sinh (\pi|a|)}, 0 \leq x \leq 2 \pi
$$

to treat the sum over the Matsubara frequencies yielding

$$
\left\langle\varphi_{\alpha,>}(x, \tau) \varphi_{\beta,>}\left(x, \tau^{\prime}\right)\right\rangle_{0,>}=\frac{K}{2} \frac{\cosh (K / 2 t-|\Delta \tau|)}{\sinh (K / 2 t)} \delta_{\alpha, \beta} \ln b,
$$

with $\Delta \tau \equiv \tau-\tau^{\prime}$.

In order to find a good gaussian approximation for $R$, we perform a variational calculation for a Sine-Gordon model:

$$
\mathcal{H}_{S G}=\mathcal{H}_{0}+\mathcal{H}_{1} \equiv \mathcal{H}_{0}-\mu \int d^{d} r \cos (\varphi(\mathbf{r})),
$$

where $\mathcal{H}_{0}$ is the gaussian part. We approximate $\mathcal{H}_{1}$ by

$$
\tilde{\mathcal{H}}_{1}=\int d^{d} r \frac{\kappa(\mathbf{r})}{2} \varphi^{2}(\mathbf{r})
$$

and define $\tilde{\mathcal{H}} \equiv \mathcal{H}_{0}+\tilde{\mathcal{H}}_{1}$. To find the optimal function $\kappa(\mathbf{r})$, the variational free energy $3^{36} \mathcal{F}_{\text {var }}$, with

$$
\mathcal{F}_{S G} \leq \mathcal{F}_{\text {var }} \equiv \tilde{\mathcal{F}}+\left\langle\mathcal{H}_{S G}-\tilde{\mathcal{H}}\right\rangle_{\tilde{\mathcal{H}}}
$$

is minimized with respect to $\kappa$ :

$$
\begin{aligned}
0=\frac{\delta \mathcal{F}_{\text {var }}}{\delta \kappa(\tilde{\mathbf{r}})} & =\beta\left\langle\mathcal{H}_{S G}-\tilde{\mathcal{H}}\right\rangle_{\tilde{\mathcal{H}}}\left\langle\frac{\varphi^{2}(\tilde{\mathbf{r}})}{2}\right\rangle_{\tilde{\mathcal{H}}}-\beta\left\langle\left(\mathcal{H}_{S G}-\tilde{\mathcal{H}}\right) \frac{\varphi^{2}(\tilde{\mathbf{r}})}{2}\right\rangle_{\tilde{\mathcal{H}}} \\
& =\frac{\beta}{2} \int d^{d} r\langle\kappa(\mathbf{r})-\mu \cos (\varphi(\mathbf{r}))\rangle_{\tilde{\mathcal{H}}}\left\langle\left\langle\varphi^{2}(\mathbf{r}) \varphi^{2}(\tilde{\mathbf{r}})\right\rangle\right\rangle_{\tilde{\mathcal{H}}} .
\end{aligned}
$$

For the last equality we took into account that the averages are gaussian such that we could apply the Wick theorem. From (A8) we finally get

$$
\kappa(\mathbf{r})=\mu\langle\cos (\varphi(\mathbf{r}))\rangle_{\tilde{\mathcal{H}}}=\mu \exp \left(-\frac{1}{2}\left\langle\varphi^{2}(\mathbf{r})\right\rangle_{\tilde{\mathcal{H}}}\right) .
$$

For small disorder A9 yields for $R\left[\Delta \varphi_{<}\right]$the approximate expression

$$
R\left[\Delta \varphi_{<}\right] \simeq-\frac{p^{2}}{2}\left(\Delta \varphi_{<}\right)^{2} e^{-\frac{p^{2}}{2}\left\langle\left(\Delta \varphi_{<}\right)^{2}\right\rangle_{0,<}} .
$$


The same result can be obtained in terms of an operator product expansion ${ }^{38}$ of $R[f]$. In order to get a RG correction to $K$ a gradient expansion of $\left(\Delta \varphi_{<}\right)^{2}$ in A10 in small $\Delta \tau$ is performed, which is justified by the exponential decay of the correlation function (A4) on the integration interval such that higher orders in $\Delta \tau$ do not contribute to the RG correction: $\left(\Delta \varphi_{<}\right)^{2} \approx\left(\partial_{T} \varphi_{<}(x, T) \Delta \tau\right)^{2}$ with $T=\left(\tau+\tau^{\prime}\right) / 2$.

The pair correlation function in the argument of the exponential function in A10 can be approximated by the expression already shown in (55), i.e.,

$$
\left\langle(\varphi(x, \tau)-\varphi(0,0))^{2}\right\rangle_{0} \simeq \frac{K}{2} \ln \left(1+\left(\frac{K}{2 \pi t}\right)^{2}\left[\cosh \left(\frac{2 \pi t x}{K}\right)-\cos \left(\frac{2 \pi t \tau}{K}\right)\right]\right) .
$$

After integration of the fast modes of $\varphi$, one rescales the system to maintain the fluctuation strength and the spatial density of the degrees of freedom $(l=\ln b)$ :

$$
\begin{aligned}
& x \rightarrow x^{\prime}=x b^{-1} \\
& \tau \rightarrow \tau^{\prime}=\tau b^{-z}, \quad T \rightarrow T^{\prime}=T b^{z} \\
& \varphi \rightarrow \varphi^{\prime}=\varphi b^{-\zeta}
\end{aligned}
$$

which leads to rescaled parameters. For our model these are given by (here in $d$ dimensions)

$$
\begin{aligned}
c^{\prime} & =c b^{d+z-2+2 \zeta}, \\
v^{\prime} & =v b^{z-1} \\
K^{\prime} & =K b^{1-d-2 \zeta} \\
t^{\prime} & =t b^{2-d-2 \zeta} \\
u^{\prime} & =u b^{2-d / 2} \\
\sigma^{\prime} & =\sigma b^{2-d} \\
w^{\prime} & =w b^{2 d}
\end{aligned}
$$

Due to the invariance of the system under a phase shift of $2 n \pi, \zeta$ is zero for symmetry reasons.

The RG contribution to the flow equation for $u^{2}$ follows from the first term of (A3) and (A4) with $\Delta \tau=0$ :

$$
u^{\prime 2}=u^{2}\left(1-\frac{p^{2} K}{2} \operatorname{coth} \frac{K}{2 t} \ln b\right) .
$$

Together with $(d=1)$ one gets

$$
\frac{u^{\prime 2}-u^{2}}{d l}=\left(3-\frac{p^{2} K}{2} \operatorname{coth} \frac{K}{2 t}\right) u^{2} .
$$

The RG correction to $K$ follows from the second term of A3) with A4 and (A10):

$$
\begin{array}{r}
K^{\prime}=K\left(1-\frac{p^{4}}{2} u^{2} \operatorname{coth} \frac{K}{2 t} \int_{0}^{K /(2 t)} d \tau \tau^{2} \frac{\cosh \left(\frac{K}{2 t}-\tau\right)}{\cosh \frac{K}{2 t}} \times\right. \\
\left.e^{-\frac{p^{2}}{2}\left\langle(\varphi(0, \tau)-\varphi(0,0))^{2}\right\rangle_{0} \ln b}\right), \quad \text { (A15) }
\end{array}
$$

and the flow equation (for $d=1+\epsilon$ ) follows from (A12c):

$$
\frac{K^{\prime}-K}{d l}=\left[-\epsilon-\frac{p^{4}}{2} u^{2} \operatorname{coth} \frac{K}{2 t} B_{0}\left(p^{2} K, \frac{K}{2 t}\right)\right] K
$$

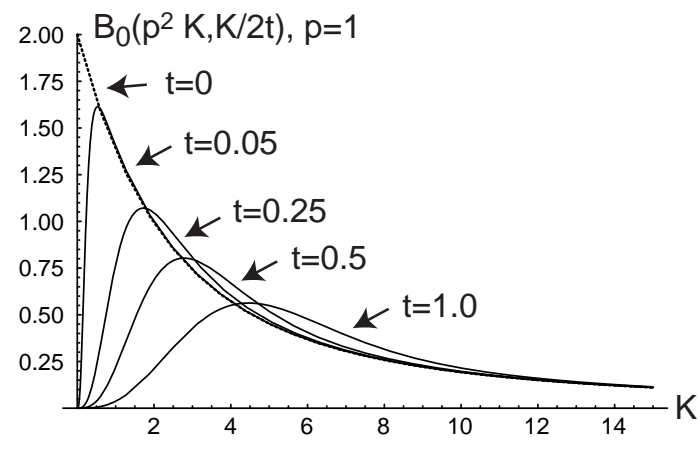

FIG. 5: Function $B_{0}\left(p^{2} K, \frac{K}{2 t}\right)$ plotted with respect to $K$ for different temperatures.

with $B_{0}$ given in (20), for which we used A11).

The function $B_{0}$ which appears in this flow equation is plotted in Fig. 5

For completeness we also plot the functions $B_{1}$ and $B_{2}$ in the relevant $K$-region for the lattice unpinning transition [see eqs. (72a) and (72b)]. Note, that for evaluating these functions at zero temperature, one has to execute the integrals at finite temperature first and then take the limit $t \rightarrow 0$.

\section{APPENDIX B: STRONG PINNING}

To calculate the phase correlation function in the strong pinning limit, it is necessary to study the order statistics of the impurity distances $\epsilon_{i}=\left(x_{i}-x_{i-1}\right)-l_{i m p}$. Following David 37 , we obtain for the probability density function (pdf) of the $\epsilon_{i}$ 's in the case of uniformly distributed impurity positions $0 \leq x_{i} \leq L: p\left(\epsilon_{i}\right)=$ $l_{\text {imp }}^{-1}\left(1-\frac{1}{L}\left(\epsilon_{i}+l_{i m p}\right)\right)^{N-1}$ with $-l_{\text {imp }} \leq \epsilon_{i} \leq L-l_{\text {imp }}$. In the thermodynamic limit the pdf can be rewritten as

$$
p\left(\epsilon_{i}\right) \approx \frac{l_{i m p}^{-1}}{e} e^{-l_{i m p}^{-1} \epsilon_{i}}, \quad-l_{i m p} \leq \epsilon_{i}<\infty .
$$

With this, one can calculate the $n$-th moment $\overline{\epsilon_{i}^{n}}$ (for 


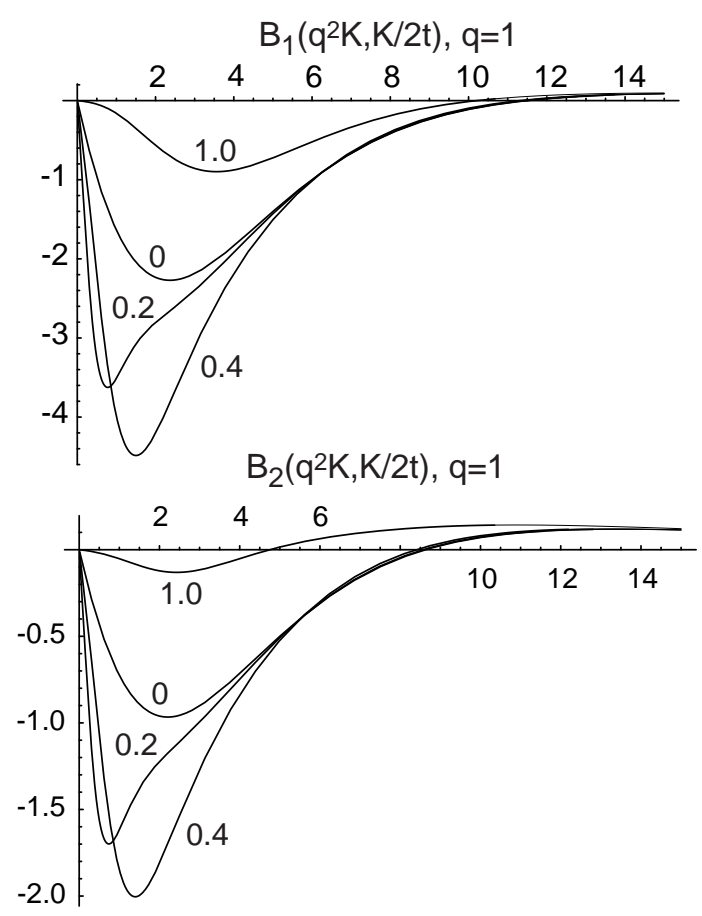

FIG. 6: Function $B_{1}\left(q^{2} K, \frac{K}{2 t}\right)$ and $B_{2}\left(q^{2} K, \frac{K}{2 t}\right)$ plotted with respect to $K$ for different temperatures, written next to the graphs, and $q=1$.

$n>1, \overline{\epsilon_{i}}=0$ ) as follows:

$$
\begin{aligned}
\overline{\epsilon_{i}^{n}} & =\int_{-l_{i m p}}^{\infty} \frac{l_{i m p}^{-1}}{e} e^{-l_{i m p}^{-1} \epsilon_{i}} \epsilon_{i}^{n} d \epsilon_{i} \\
& =\left.\frac{l_{i m p}^{-1}}{e}(-1)^{n} \frac{\partial^{n}}{\partial \lambda^{n}}\right|_{\lambda=l_{i m p}^{-1}} \frac{e^{\lambda l_{i m p}}}{\lambda}
\end{aligned}
$$

Using $\left.\frac{\partial^{n}}{\partial x^{n}}\right|_{x=1} \frac{e^{x-1}}{x}=(-1)^{n} n !\left(\sum_{k=1}^{n} \frac{(-1)^{k}}{k !}+1\right)$ yields: $\overline{\epsilon_{i}^{n}}=\frac{n !}{c^{n}} \sum_{k=2}^{n} \frac{(-1)^{k}}{k !}$ and for the correlator $\overline{\epsilon_{i} \epsilon_{j}}=$ $l_{i m p}^{2} \delta_{i j}$.

With this results we can derive the pair correlation function (63). Therefore we calculate the discrete version $\overline{\left\langle\left(\varphi_{n}-\varphi_{1}\right)^{2}\right\rangle}$ in the limit $T \rightarrow 0$. With the definitions given below eq. (36), we can rewrite:

$$
\left(\varphi_{n}-\varphi_{1}\right)^{2}=\frac{4 \pi^{2}}{p^{2}}\left(\sum_{i=1}^{n-1}\left(h_{i}-\frac{p Q \epsilon_{i}}{2 \pi}-\gamma\right)\right)^{2}
$$

Using $\overline{\epsilon_{i}}=\overline{h_{i}}=0$ and $\overline{h_{i} h_{j}} \propto \delta_{i j}$ leads to

$$
\overline{\left\langle\left(\varphi_{n+1}-\varphi_{1}\right)^{2}\right\rangle}=\frac{4 \pi^{2}}{p^{2}} \underbrace{\left\langle\left(h_{i}-\frac{p Q \epsilon_{i}}{2 \pi}-\gamma\right)^{2}\right\rangle}_{\equiv \tilde{\mathrm{C}}} \cdot n .
$$

Because only the value $\left[\frac{p Q \epsilon_{i}}{2 \pi}+\gamma\right]$ for $h_{i}$ is taken into account for evaluation of the thermal average, we get

$$
\begin{aligned}
& \tilde{\mathrm{C}}=\overline{\left(\frac{p Q \epsilon_{i}}{2 \pi}+\gamma-\left[\frac{p Q \epsilon_{i}}{2 \pi}+\gamma\right]\right)^{2}} \\
& =\int_{-l_{i m p}}^{\infty} d \epsilon_{i} \frac{l_{i m p}^{-1}}{e} e^{-l_{i m p}^{-1} \epsilon_{i}}\left(\frac{p Q \epsilon_{i}}{2 \pi}+\gamma-\left[\frac{p Q \epsilon_{i}}{2 \pi}+\gamma\right]\right)^{2} .
\end{aligned}
$$

If we substitute $x=l_{i m p}^{-1} \epsilon_{i}+1$ and take into account that $[x+n]=[x]+n$ for $n \in \mathbb{Z}$, we get

$$
\tilde{\mathrm{C}}=\int_{0}^{\infty} d x e^{-x}\left(\frac{x}{2 \alpha}-\left[\frac{x}{2 \alpha}\right]\right)^{2}
$$

with the parameter $\alpha=\frac{\pi}{p Q l_{i m p}}$. Now the quadratic term in the integral is expanded, which leads to the following three (converging) integrals

$$
\begin{aligned}
I_{1} & \equiv \frac{1}{4 \alpha^{2}} \int_{0}^{\infty} d x e^{-x} x^{2}=\frac{1}{2 \alpha^{2}} \\
I_{2} & \equiv-\frac{1}{\alpha} \int_{0}^{\infty} d x e^{-x} x\left[\frac{x}{2 \alpha}\right] \\
& =-\frac{1}{\alpha} \sum_{k=1}^{\infty} \int_{(2 k-1) \alpha}^{(2 k+1) \alpha} d x e^{-x} x\left[\frac{x}{2 \alpha}\right] \\
I_{3} & \equiv \int_{0}^{\infty} d x e^{-x}\left[\frac{x}{2 \alpha}\right]^{2}, \\
& =\sum_{k=1}^{\infty} \int_{(2 k-1) \alpha}^{(2 k+1) \alpha} d x e^{-x}\left[\frac{x}{2 \alpha}\right]^{2}
\end{aligned}
$$

with $\tilde{\mathrm{C}}=I_{1}+I_{2}+I_{3}$. For $(2 k-1) \alpha \leq x \leq(2 k+1) \alpha$, $k \in \mathbb{Z},\left[\frac{x}{2 \alpha}\right]=k$, such that the Gaussian brackets in the finite integrals in $I_{2}$ and $I_{3}$ can be replaced by $k$ or $k^{2}$, respectively:

$$
\begin{aligned}
& I_{2}=-\frac{1}{\alpha} \sum_{k=1}^{\infty} k \int_{(2 k-1) \alpha}^{(2 k+1) \alpha} d x e^{-x} x, \\
& I_{3}=\sum_{k=1}^{\infty} k^{2} \int_{(2 k-1) \alpha}^{(2 k+1) \alpha} d x e^{-x} .
\end{aligned}
$$

The values of these two simple integrals are

$$
\begin{array}{cc}
\int_{(2 k-1) \alpha}^{(2 k+1) \alpha} d x e^{-x} x=2 e^{-2 k \alpha}((1+2 k \alpha) \sinh (\alpha)- & \alpha \cosh (\alpha)), \\
\int_{(2 k-1) \alpha}^{(2 k+1) \alpha} d x e^{-x} & =2 e^{-2 k \alpha} \sinh (\alpha) .
\end{array}
$$

The remaining sums in $I_{2}$ and $I_{3}$ are only derivatives of the geometric series which can be easily evaluated. 
The result is

$$
\begin{aligned}
\tilde{\mathrm{C}} & =\frac{1}{2 \alpha}\left(\frac{1}{\alpha}-\frac{1}{\sinh (\alpha)}\right) \\
& =\frac{1}{12}-\frac{7}{720} \alpha^{2}+\frac{31}{30240} \alpha^{4}+\mathcal{O}\left(\alpha^{6}\right),
\end{aligned}
$$

where the expansion is useful only if $\alpha$ is small, i.e., $Q l_{\text {imp }} \gg 1$. If we would have neglected the order statistics of the impurity distances we would get only the leading constant: $\tilde{\mathrm{C}}=1 / 12$.

(B8) yields the presented expression for the pair correlation function (63).

\section{APPENDIX C: CORRELATION LENGTH IN THE CLASSICAL REGION AT FINITE TEMPERATURE}

In the weak pinning limit, $1 \ll l_{i m p} Q \ll c /\left(U_{i m p} \rho_{1}\right)$ or $L_{F L} \gg l_{i m p}$, the classical Hamiltonian can be rewritten to a random-field $X Y$-model

$$
\begin{aligned}
\mathcal{H}_{\text {class }}(L)= & \int_{0}^{L} d x\left\{\frac{c}{2}\left(\partial_{x} \varphi(x)-\tilde{\sigma}\right)^{2}+\right. \\
& \left.\frac{U_{i m p} \rho_{1}}{l_{i m p}} \cos [p(\varphi(x)-\alpha(x))]\right\}
\end{aligned}
$$

where $\alpha(x)$ is a random phase with zero average and

$$
\overline{e^{\imath p\left(\alpha(x)-\alpha\left(x^{\prime}\right)\right)}}=l_{i m p} \delta\left(x-x^{\prime}\right) .
$$

In the following we consider only the backward scattering term of the correlation function $C_{b}$ and therefore neglect the forward scattering term $\tilde{\sigma}=U_{i m p} f /\left(c \pi l_{i m p}\right)$.

The goal is now to find an effective temperature, which replaces $T$ in the correlation function for the free case (64). We start with a Burgers-like equation, which one gets after a Cole-Hopf transformation from the transfer matrix equation, for the restricted free energy $\mathcal{F}(x, \varphi)=$
$-T \ln \mathcal{Z}(x, \varphi)$ with the partition function

$$
\mathcal{Z}(x, \varphi) \equiv \int_{\varphi(0)}^{\varphi(x)=\varphi} \mathcal{D} \varphi e^{-H(x) / T}
$$

The equation reads

$$
\frac{\partial \mathcal{F}}{\partial x}=\frac{T}{2 c} \frac{\partial^{2} \mathcal{F}}{\partial \varphi^{2}}-\frac{1}{2 c}\left(\frac{\partial \mathcal{F}}{\partial \varphi}\right)^{2}+\underbrace{\frac{U_{i m p} \rho_{1}}{l_{i m p}} \cos [p(\varphi(x)-\alpha(x))]}_{U(x, \varphi)} .
$$

Using the Fourier transform $\mathcal{F}(x, \varphi)=$ $\int \frac{d k d \omega}{(2 \pi)^{2}} e^{\imath(\omega \varphi-k x)} \mathcal{F}(k, \omega)$ (analogous for $U(x, \varphi)$ ), (C3) is rewritten as

$$
\begin{gathered}
-\imath k \mathcal{F}(k, \omega)=-\frac{T \omega^{2}}{2 c} \mathcal{F}(k, \omega)+U(k, \omega)+ \\
\frac{1}{2 c} \int \frac{d k^{\prime} d \omega^{\prime}}{(2 \pi)^{2}} \omega^{\prime}\left(\omega-\omega^{\prime}\right) \mathcal{F}\left(k-k^{\prime}, \omega-\omega^{\prime}\right) \mathcal{F}\left(k^{\prime}, \omega^{\prime}\right),
\end{gathered}
$$

with

$$
\begin{aligned}
& U(k, \omega)=\frac{\pi U_{i m p} \rho_{1}}{l_{i m p}}\left\{h_{+}(k) \delta(\omega-p)\right.\left.+h_{-}(k) \delta(\omega+p)\right\}, \\
& h_{ \pm}(k) \equiv \int d x e^{\imath[k x \mp p \alpha(x)]} .
\end{aligned}
$$

Introduction the dimensionless quantities

$$
\begin{aligned}
g_{0}(k, \omega) & =\frac{1}{\pi t \omega^{2} / 2-\imath k / \Lambda}, \\
\epsilon & =\frac{U_{i m p} \rho_{1}}{l_{i m p} \Lambda^{2} c}, \\
u(k, \omega) & =(\epsilon \Lambda c)^{-1} U(k, \omega),
\end{aligned}
$$

and setting $\mathcal{F}(k, \omega) \equiv c \epsilon g(k, \omega) u(k, \omega)$ we obtain the following, self-consistent equation for the Green's function $g(k, \omega)$ :

$$
g(k, \omega) u(k, \omega)=g_{0}(k, \omega) u(k, \omega)+\frac{\epsilon}{2} g_{0}(k, \omega) \int \frac{d k^{\prime} d \omega^{\prime}}{\Lambda(2 \pi)^{2}} \omega^{\prime}\left(\omega-\omega^{\prime}\right) g\left(k-k^{\prime}, \omega-\omega^{\prime}\right) g\left(k^{\prime}, \omega^{\prime}\right) u\left(k-k^{\prime}, \omega-\omega^{\prime}\right) u\left(k^{\prime}, \omega^{\prime}\right)
$$

For $\epsilon<1$, i.e., for weak disorder, this equation is iterated to first non-vanishing order in $\epsilon$ (one-loop approximation) and averaged over disorder. The disorder average $\overline{u(k, \omega) u\left(k^{\prime}, \omega^{\prime}\right)}$ can be calculated using (C5) and (C2), which gives

$$
\begin{aligned}
\overline{u(k, \omega) u\left(k^{\prime}, \omega^{\prime}\right)} & =\Lambda^{2} \pi^{2}\left\{\overline{h_{+}(k) h_{-}\left(k^{\prime}\right)} \delta(\omega-p) \delta\left(\omega^{\prime}+p\right)+\overline{h_{-}(k) h_{+}\left(k^{\prime}\right)} \delta(\omega+p) \delta\left(\omega^{\prime}-p\right)\right\} \\
& =2 \Lambda^{2} l_{i m p} \pi^{3} \delta\left(k+k^{\prime}\right) \delta\left(\omega+\omega^{\prime}\right)\{\delta(\omega+p)+\delta(\omega-p)\} \\
& \equiv 2 \delta\left(k+k^{\prime}\right) \delta\left(\omega+\omega^{\prime}\right) D(\omega, k) .
\end{aligned}
$$


Therefore, we get for $g$ in order $\epsilon^{2}$

$$
g(k, \omega)=g_{0}(k, \omega)+\epsilon^{2} g_{0}^{2}(k, \omega) \int \frac{d k^{\prime} d \omega^{\prime}}{\Lambda^{2}(2 \pi)^{4}}\left(\omega-\omega^{\prime}\right) \omega^{\prime} \omega\left(-\omega^{\prime}\right) g_{0}\left(k^{\prime}, \omega^{\prime}\right) g_{0}\left(k-k^{\prime}, \omega-\omega^{\prime}\right) g_{0}\left(-k^{\prime},-\omega^{\prime}\right) D\left(k^{\prime}, \omega^{\prime}\right) .
$$

(a)

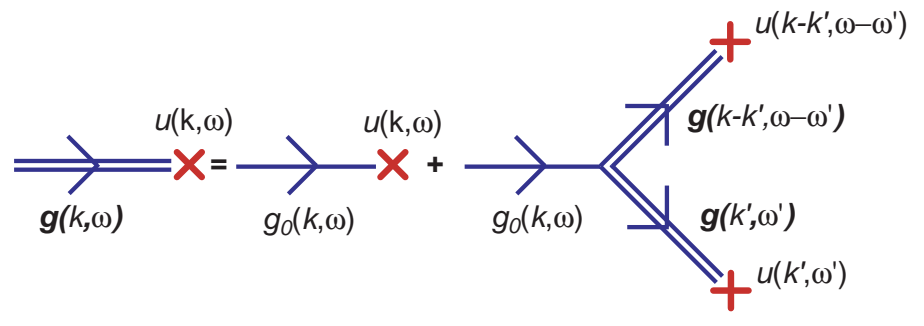

(b)

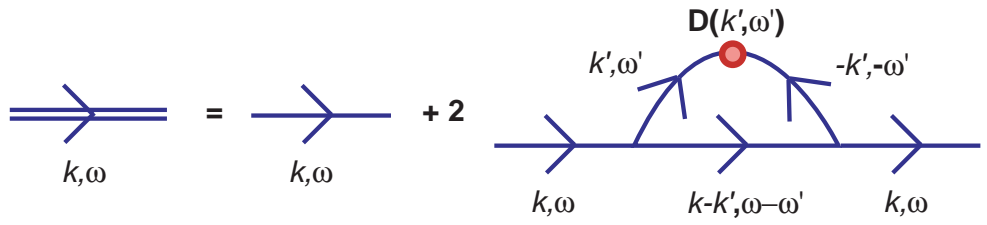

FIG. 7: Diagrams for (a) eq. C9. and (b) eq. C11.

The diagrams visualizing eqs. [C9] and [C11) are depicted in figure 7

For $k=0$ (C11) reduces to

$g(0, \omega)=g_{0}(0, \omega)\left[1+\epsilon^{2} \frac{p^{2} l_{i m p}}{8 \pi t \omega} \int d k^{\prime} \frac{1}{\left(k^{\prime} / \Lambda\right)^{2}+\left(\pi p^{2} t / 2\right)^{2}}\left\{\frac{p-\omega}{\pi t(\omega-p)^{2} / 2-\imath k^{\prime} / \Lambda}-\frac{p+\omega}{\pi t(\omega+p)^{2} / 2-\imath k^{\prime} / \Lambda}\right\}\right]$

Because we calculate the correlation length in the thermal regime (see figure 31) with $t \gtrsim t_{u}$ the $k^{\prime}$-integral in (C12) gives the biggest contribution to $g$ at small $\omega$. In this case the $k^{\prime}$-integral can be easily calculated which leads to

$$
g(0, \omega \ll 1) \approx g_{0}(0, \omega)\left(1-\epsilon^{2} \frac{\Lambda l_{i m p}}{2 \pi^{3} p^{2} t^{3}}\right)
$$

or for the effective Temperature $T_{\text {eff }}$

$$
\frac{1}{T_{\text {eff }}} \approx \frac{1}{T}\left(1-\epsilon^{2} \frac{\Lambda l_{i m p}}{2 \pi^{3} p^{2} t^{3}}\right)=\frac{1}{T}\left(1-\frac{1}{2}\left(\frac{t_{u}}{\pi p^{2} t}\right)^{3}\right)
$$

which yields for the correlation length

$$
\xi^{-1} \approx \frac{\pi}{2} f(T) t\left[1+\frac{1}{2}\left(\frac{t_{u}}{\pi p^{2} t}\right)^{3}\right] \Lambda
$$

as written in the text. For high temperatures $t \gg t_{u}$ we recover the linear $t$ dependency of the free case.

A related calculation for directed polymers and interface growth can be found in [39]. Note, that in this paper $x$ plays the role of $\varphi$ and $t$ the role of $x$ in the above calculation.

TABLE III: List of used quantities

\begin{tabular}{clr}
\hline \hline symbol & quantity & eq. ref. \\
\hline$a$ & lattice constant & \\
$b=e^{-l}$ & rescaling parameter of order 1 & (20) \\
$B_{i}$ & functions used in flow equations & (4a) \\
$c$ & elastic constant & (52), (59) \\
$C(x, \tau), C_{f}, C_{b}$ & phase correlation functions
\end{tabular}


TABLE III: (continued...)

\begin{tabular}{|c|c|c|}
\hline symbol & quantity & eq. ref. \\
\hline$d$ & spatial dimension & \\
\hline$f(T)$ & condensate density & (4) \\
\hline $\mathcal{F}$ & free energy & 15 \\
\hline$g$ & dimensionless electron-phonon coupling constant & (4) \\
\hline$g_{i}$ & functions used in $B_{i}$ & (20) \\
\hline$g_{q}$ & electron-phonon coupling constants & (2) \\
\hline$h_{i}$ & integers (cf. strong pinning) & 38 \\
\hline$\hat{\mathcal{H}}, \hat{\mathcal{H}}_{0}$ & Hamiltonian (complete and free) & (4) \\
\hline$k, k_{n}$ & wave vectors & \\
\hline$k_{F}$ & Fermi wave vector & \\
\hline$K, K(l), K_{0}$ & dimensionless parameter for quantum fluctuations & $8 \mathrm{~b}, 19 \mathrm{~b}$ \\
\hline$K_{u}, K_{u}^{*}$ & $K$-values defining the separatrix/fixed point of the disorder unpinning transition & (27) \\
\hline$K_{w}, K_{w}^{*}$ & $K$-values defining the separatrix/fixed point of the lattice unpinning transition & 74 \\
\hline$L$ & system length & (4) \\
\hline$L_{F L}$ & Fukuyama-Lee length & (7) \\
\hline$l_{i m p}$ & mean impurity distance & (5) \\
\hline $\mathcal{L}_{0}$ & free (gaussian) part of the Lagrangian & (16) \\
\hline$n_{i}$ & integers (cf. strong pinning) & (37) \\
\hline$N_{\text {imp }}$ & number of impurities & $4 \mathrm{~b}$ \\
\hline$p$ & commensurability used in the density & (1) \\
\hline$p\left(\epsilon_{i}\right)$ & probability density function of $\epsilon_{i}$ & (61) \\
\hline$\hat{P}$ & momentum operator, conjugate to $\hat{\varphi}$ & (4) \\
\hline$Q$ & density wave vector & (1) \\
\hline$q$ & commensurability used in the lattice potential & (4c) \\
\hline $\mathcal{S}, \mathcal{S}_{0}$ & action (full and gaussian part) & (18) \\
\hline $\mathcal{S}^{(n)}$ & replicated action & (16) \\
\hline $\mathcal{S}_{S F}, \mathcal{S}_{L P}$ & action for superfluids and lattice potential, respectively & 70 \\
\hline$S, S_{1}$ & density correlation functions & (48), 49, \\
\hline$T$ & temperature & \\
\hline$T_{c}^{M F}$ & mean-field condensation temperature & \\
\hline$t, t(l), t_{0}$ & parameter for thermal fluctuations & $8 \mathrm{a}$ \\
\hline$t_{u}=1 /\left(\Lambda L_{F L}\right)$ & crossover temperature from classical disordered to thermal regime & \\
\hline$t_{K}$ & temperature separating the thermal and disordered regime & \\
\hline$U(x)$ & disorder potential & $4 \mathrm{~b}$ \\
\hline$U_{i}$ & impurity potential & $4 \mathrm{~b}$ \\
\hline$U_{i m p}$ & mean impurity potential & [5] \\
\hline$u, u(l), u_{0}$ & dimensionless parameter for disorder fluctuations & $8 \mathrm{c}$ \\
\hline$v_{F}$ & Fermi velocity & \\
\hline$v$ & phason velocity & (4) \\
\hline$V_{c}(x)$ & Coulomb potential & 10 \\
\hline$W$ & lattice potential strength & $4 \mathrm{c}$ \\
\hline$w, w(l), w_{0}$ & dimensionless parameter for lattice potential strength & $8 \mathrm{~d}$ \\
\hline$x_{i}$ & impurity positions & $4 \mathrm{~b}$ \\
\hline$z$ & dimensionless distance in $\tau$-x-space & \\
\hline$\alpha$ & parameter used in the strong pinning limit & 63 \\
\hline$\beta$ & inverse temperature & \\
\hline$\gamma$ & parameter for KT flow equations & $28 \mathrm{a}$ \\
\hline$\Delta$ & order parameter & 2 \\
\hline$\epsilon_{i}$ & deviation from mean impurity distance & 38 \\
\hline$\zeta$ & transverse width of the quasi one-dimensional system & \\
\hline$\eta, \tilde{\eta}$ & $=B_{0}\left(p^{2} K_{u}^{*}, \infty\right),=-B_{2}\left(q^{2} / K_{w}^{*}, \infty\right)$, respectively & \\
\hline$\lambda$ & density wave length & \\
\hline$\lambda_{T}$ & de Broglie wave length & (9) \\
\hline
\end{tabular}


TABLE III: (continued...)

\begin{tabular}{clr}
\hline symbol & quantity & eq. ref. \\
\hline$\Lambda$ & momentum cutoff \\
$\xi, \xi_{u}, \xi_{w}$, etc. & correlation lengths \\
$\rho(x), \rho_{S F}(x)$ & charge/spin or superfluid density \\
$\rho_{0}$ & mean density \\
$\rho_{1}$ & density amplitude for harmonic part of $\rho(x)$ \\
$\sigma$ & forward scattering amplitude \\
$\tau$ & imaginary time coordinate \\
$\varphi$ & phase variable \\
$\chi$ & parameter for KT flow equations \\
$\Upsilon$ & auxiliary function \\
$\omega_{n}$ & Matsubara frequencies \\
\hline \hline
\end{tabular}

1 G. Grüner, Rev. Mod. Phys. 60, 1128 (1988); Density Waves in Solids (Addison-Wesley, Reading, MA, 1994).

2 G. Grüner, Rev. Mod. Phys. 66, 1 (1994).

3 Proceedings of the International Workshop on Electronic Crystals: ECRYS-99, La Colle-sur-Loup, France, 1999, edited by S. Brazovski and P. Monceau (EDP Sciences, Les Ulis, France, 1999).

${ }^{4}$ G. Blatter, M.V. Feigel'man, V. Geshkenbein, A.I. Larkin, and V.M. Vinokur, Rev.Mod.Phys. 66, 1125 (1994).

5 T. Nattermann and S. Scheidl, Adv. Phys. 49, 607 (2000).

6 J.L. Cardy and S. Ostlund, Phys. Rev. B 25, 6899 (1982).

7 J. Villain and J.F. Fernandez, Z. Phys. B 54, 139 (1984).

8 M. Feigel'man, Sov. Phys. JETP 52, 555 (1980).

9 A. Glatz and M.S. Li, Phys. Rev. B 64, 184301 (2001).

${ }^{10}$ H. Fukuyama, Lect. Notes Phys. 217, 487 (1984).

11 T. Giamarchi and H.J. Schulz, Europhys. Lett. 3, 1287 (1987); Phys. Rev. B 37, 325 (1988); see also for CDWs, H.J. Schulz, Phys. Rev. Lett 71, 1864 (1993) .

12 S. Chakravarty, B.I. Halperin, and D.R. Nelson, Phys. Rev. Lett 60, 1057 (1988); Phys. Rev. B 39, 2344 (1989).

13 J.D. Brock, D.A. DiCarlo, W.J. Podulka, M. Sutton, E. Sweetland, and R.E. Thorne, J. Phys. IV 3, 115 (1993).

14 See, e.g., R.M. Bradley and S. Doniach, Phys. Rev. B 30, 1138 (1984); M.P.A. Fisher and G. Grinstein, Phys. Rev. Lett. 60, 208 (1988); S.R. Renn and J.M. Duan, Phys. Rev. Lett. 76, 3400 (1996); A.D. Zaikin et al. Phys. Rev. Lett 781552 (1997).

15 S.E. Korshunov, Sov. Phys. JETP 68, 609 (1989).

16 A. Glatz and T. Nattermann, Phys. Rev. Lett. 88, 256401 (2002).

17 R.E. Thorne, Physics Today, May 1996, 42 (1996).

18 U. Eckern and A. Geier, Z. Phys. B 65, 15 (1986); C.R. Werner and U. Eckern, Ann. Phys. 6, 595 (1997).

19 Y. Tomio and Y. Suzumura, J. Phys. Soc. Jap. 70, Iss. 10, 2884 (2001).

${ }^{20}$ K. Maki, Phys. Lett. A 202, 313 (1995).

${ }^{21}$ H. Maurey and T. Giamarchi, Phys. Rev. B 51, 10833
(1995)

${ }^{22}$ H.J. Schulz, Phys. Rev. Lett. 71, 1864 (1993).

23 B.L. Altshuler and A.G. Aronov, in Electron-Electron Interaction in Disordered Systems, ed. by A.L. Efros and M. Pollak, North-Holland, Amsterdam 1985.

24 P.A. Lee and H. Fukuyama, Phys. Rev. B 17, 542 (1978).

25 U. Schultz, J. Villain, E. Brezin, and H. Orland, J. Stat. Phys. 51, 1 (1988).

26 J.M. Kosterlitz and D.J. Thouless, J. Phys. C 6, 1181 (1974).

27 I.F. Herbut, Phys. Rev. B 58, 971 (1998).

28 T. Giamarchi and H.J. Schulz, Phys. Rev. B 39, 4620 (1989).

29 S. Sachdev, Quantum Phase Transitions (Cambridge University Press, 1999).

30 D.A. Huse, C.L. Henley, and D.S. Fisher, Phys. Rev. Lett. 55, 2924 (1985).

31 F.D.M. Haldane, Phys. Rev. Lett. 47, 1840 (1981). (note that in this Letter the meaning of $\varphi$ and $\theta$ is interchanged).

32 A. Lenard, J. Math. Phys. 2, 682 (1961).

33 R. Shankar, Int. J. Mod. Phys. B 4, 2371 (1990).

34 T. Giamarchi, P. Le Doussal, and E. Orignac, Phys. Rev. B 64, 245119 (2001).

35 K.G. Wilson and J. Kogut, Phys. Rep. 12, 75 (1973).

36 The inequality for the variational free energy follows directly from $1-\frac{1}{x} \leq \ln (x) \leq x-1$ for $x>0$, if one considers the expression $K\left(\hat{\rho}_{S G}, \hat{\tilde{\rho}}\right) \equiv \operatorname{Tr} \hat{\tilde{\rho}}\left(\ln (\hat{\tilde{\rho}})-\ln \left(\hat{\rho}_{S G}\right)\right.$, where $\hat{\rho}$ denotes the density matrix of the system.

37 H. A. David, Order Statistics, (John Wiley \& Sons Inc., 1970).

38 H.J.F. Knops and L.W.J den Ouden, Physica A 103, 597 (1980).

39 E. Medina, T. Hwa, M. Kardar, and Yi-C. Zhang, Phys. Rev. A 39, 3053 (1989). 